\title{
Convolution estimates for measures on some complex curves
}

\author{
Hyunuk Chung ${ }^{1} \cdot$ Seheon Ham ${ }^{2}$
}

Received: 1 June 2018 / Accepted: 20 October 2018 / Published online: 8 November 2018 (c) Fondazione Annali di Matematica Pura ed Applicata and Springer-Verlag GmbH Germany, part of Springer Nature 2018

\begin{abstract}
We consider the convolution operator for a measure supported on a complex curve. The measure which we consider here is an analogue of the affine arclength measure for real curves. By modifying a combinatorial argument called the band structure argument due to Christ and Stovall, we prove the (nearly) optimal Lorentz space estimates. This includes the optimal strong type estimates as special cases.
\end{abstract}

Keywords Convolution estimate Complex curves · Finite type Affine arclength measure Mathematics Subject Classification 42B10

\section{Introduction}

For $d \geq 2$, we consider 2-dimensional surfaces in $\mathbb{R}^{2 d}$ so-called complex curves in $\mathbb{C}^{d}$. Let $\Gamma(z)=\left(\Gamma_{1}(z), \ldots, \Gamma_{d}(z)\right)$ be a complex curve defined in the unit disk $D=\{z:|z|<1\}$, where $\Gamma_{i}, 1 \leq i \leq d$ are analytic functions on $D$. Then $\Gamma$ can be regarded as a 2-dimensional surface in $\mathbb{R}^{2 d}$ defined by the real mapping

$$
z=(x, y) \mapsto \Gamma(x, y)=\left(\operatorname{Re}\left(\Gamma_{1}(z)\right), \operatorname{Im}\left(\Gamma_{1}(z)\right), \ldots, \operatorname{Re}\left(\Gamma_{d}(z)\right), \operatorname{Im}\left(\Gamma_{d}(z)\right)\right) .
$$

We consider the convolution operator along $\Gamma$ given by

$$
\mathcal{A} f(x)=\int_{D} f(x-\Gamma(z)) W_{\Gamma}(z) \mathrm{d} \mu(z),
$$

Seheon Ham is supported in part by NRF-2017R1C1B2002959 (Republic of Korea).

$凶$ Seheon Ham

seheonham@snu.ac.kr

Hyunuk Chung

nuki@postech.ac.kr

1 Department of Mathematics, Pohang University of Science and Technology, Pohang 37673, Republic of Korea

2 Research institute of Mathematics, Seoul National University, Seoul 08826, Republic of Korea 
where

$$
W_{\Gamma}(z) \mathrm{d} \mu(z)=\left|\operatorname{det}\left(\Gamma^{\prime}(z) \Gamma^{\prime \prime}(z) \cdots \Gamma^{(d)}(z)\right)\right|^{\frac{4}{d(d+1)}} \mathrm{d} \mu(z)
$$

and $\mathrm{d} \mu(z)=\mathrm{d} \mu(\Gamma(z))=\mathrm{d} x \mathrm{~d} y$ is the induced Lebesgue measure on $\Gamma$. Note that $W_{\Gamma}(z) \mathrm{d} \mu(z)$ is an analogue of the affine arclength measure in the case of real curves.

It is obvious that $\mathcal{A}: L^{\infty}\left(\mathbb{R}^{2 d}\right) \rightarrow L^{\infty}\left(\mathbb{R}^{2 d}\right)$, and by duality $\mathcal{A}: L^{1}\left(\mathbb{R}^{2 d}\right) \rightarrow L^{1}\left(\mathbb{R}^{2 d}\right)$. By interpolating these trivial estimates, it follows that $\mathcal{A}: L^{p} \rightarrow L^{p}$ for all $1 \leq p \leq \infty$.

We are concerned with how much the integrability can be improved via the measure $W_{\Gamma}(z) \mathrm{d} \mu(z)$. Moreover, we will obtain a near-optimal estimate in Lorentz spaces, that is

$$
\|\mathcal{A} f\|_{L^{q_{d}, v}\left(\mathbb{R}^{2 d}\right)} \leq C\|f\|_{L^{p_{d}, u}\left(\mathbb{R}^{2 d}\right)} \text { for } u<q_{d}, p_{d}<v \text {, and } u<v .
$$

Here $p_{d}=\frac{d+1}{2}$ and $q_{d}=\frac{d(d+1)}{2(d-1)}$.

For $d=2$, i.e., $\Gamma(z)=(z, \phi(z))$, Drury and Guo [18] obtained (2) for $q_{3}=v=3$ and $p_{3}=u=3 / 2$ under some nondegeneracy conditions on $\phi(z)$.

In this paper, we prove (2) for certain types of complex curves in $\mathbb{C}^{d}$ for $d \geq 3$.

Theorem 1 For $d=3$, let $\phi_{d}$ be a polynomial of degree at most $N$, and for $d \geq 4$, let $\phi_{d}:=$ $z^{N}$. Then there exists a constant $C=C(d, N)$ such that (2) holds for $\Gamma=\left(z, \ldots, z^{d-1}, \phi_{d}\right)$.

By interpolation with the trivial $L^{p}-L^{p}$ estimate, Theorem 1 implies that $\mathcal{A}$ is of strong type for $(1 / p, 1 / q) \in \mathcal{R}$, where $\mathcal{R}$ is the closed trapezoid with vertices at $(0,0)$, $(1,1),\left(1 / p_{d}, 1 / q_{d}\right)$, and $\left(1 / q_{d}^{\prime}, 1 / p_{d}^{\prime}\right)$ in the plane. (We will discuss sharpness of (2) in "Appendix A".)

In view of the uniformity of $C(d, N)$, one can see that (2) holds for $1 / p=1 / q+2 / d(d+1)$ and $q_{d}^{\prime} \leq p \leq p_{d}$ when the domain of $\Gamma$ is all of $\mathbb{C}$.

When $d=3$, we also prove that (2) holds for small perturbations of monomials and hence locally for all finite type curves. Let $\mathbf{a}=\left(a_{1}, \ldots, a_{d}\right)$ denote $d$-tuple of positive integers satisfying $1 \leq a_{1}<a_{2}<\cdots<a_{d}$. For a given $\epsilon>0$, let us set a class of curves given by

$$
\mathcal{G}^{\mathbf{a}}(\epsilon)=\left\{\Gamma(z)=\left(z^{a_{1}} \psi_{1}(z), z^{a_{2}} \psi_{2}(z), \ldots, z^{a_{d}} \psi_{d}(z)\right):\left\|\psi_{i}-1\right\|_{C^{a_{d}+1}(2 D)} \leq \epsilon\right\},
$$

where $\psi_{j}$ 's are complex-valued functions, $2 D=\{|z|<2\}$, and $\|f\|_{C^{k}(D)}=$ $\sum_{\ell=0}^{k} \sup _{z \in D}\left|f^{(\ell)}(z)\right|$.

Theorem 2 Let $d=3$, and suppose $\Gamma \in \mathcal{G}^{\mathbf{a}}(\epsilon)$. If $\epsilon>0$ is sufficiently small, there exists a constant $C>0$, depending only on a, such that (2) holds.

Remark 1 Unlike the case of real curves, the constant $C$ in Theorem 2 depends on a even for monomial curves. In fact, when $\epsilon=0$, i.e., $\Gamma(z)$ is a monomial curve such that $\left|a_{j}\right| \leq N$ for $1 \leq j \leq 3$, then the constant $C$ depends on the choice of $\mathbf{a}=\left(a_{1}, a_{2}, a_{3}\right)$, but it actually depends only on $N$. This is because in our argument we decompose the complex plane into thin sectors in order to control the modulus of a complex number with its real part. Here, the angles of sectors depend on the $a_{j}$ 's.

Now let us recall some results for real curves in $\mathbb{R}^{d}$. Let $\gamma(t): I=[0,1] \rightarrow \mathbb{R}^{d}$ be a smooth space curve in $\mathbb{R}^{d}$. Let us denote the affine arclength measure along $\gamma(t)$ by

$$
w(t) \mathrm{d} t=\left|\operatorname{det}\left(\gamma^{\prime}(t) \gamma^{\prime \prime}(t) \cdots \gamma^{(d)}(t)\right)\right|^{\frac{2}{d(d+1)}} \mathrm{d} t .
$$

There has been much work about estimates for the convolution operator given by

$$
\mathcal{B} f(x)=\int_{I} f(x-\gamma(t)) w(t) \mathrm{d} t .
$$


Let $\gamma(t)=\left(t, t^{2}, \ldots, t^{d}\right)$. When $d=2$, Littman [24] proved the strong type $(3 / 2,3)$ for $\mathcal{B}$. Oberlin $[25,26,28,29]$ established the strong type $(2,3)$ for $d=3$ and the restricted weak type $(5 / 2,10 / 3)$ for $d=4$. The endpoint case in $d=2$, i.e., $L^{3 / 2, r}\left(\mathbb{R}^{2}\right) \rightarrow L^{3, r}\left(\mathbb{R}^{2}\right)$ estimate for $3 / 2 \leq r \leq 3$, was shown by Bak, Oberlin and Seeger [2]. The estimate $L^{2}\left(\mathbb{R}^{3}\right) \rightarrow$ $L^{3 / 2,2}\left(\mathbb{R}^{3}\right.$ ) (which is the case $u=v=p_{3}=2$ ) was obtained by Bennett and Seeger in [6], where the result was proved by analyzing the singularities of the phase function of a certain oscillatory integral operator. When $d \geq 2$, Christ [10] proved the restricted weak type $\left(p_{d}, q_{d}\right)$ for $\mathcal{B}$ by using the band structure argument. This argument was extended by Stovall [33] to establish the $L^{p_{d}, u} \rightarrow L^{q_{d}, v}$ estimates for $u<q_{d}, v>p_{d}$, and $u<v$.

Gressman [22] proved the restricted weak type $\left(p_{\mathbf{a}}, q_{\mathbf{a}}\right)$ for a class of monomial curves $\gamma(t)=\left(t^{a_{1}}, \ldots, t^{a_{d}}\right)$ for positive integers $a_{1}<\cdots<a_{d}$, and for $w(t)=|t|^{b},-1<b \leq 0$. Here, $\left(p_{\mathbf{a}}, q_{\mathbf{a}}\right)$ depends on the exponents $a_{1}, \ldots, a_{d}$, where $\mathbf{a}=\left(a_{1}, \ldots, a_{d}\right)$. See also $[8,27,32]$ for 3-dimensional monomial curves.

Drury [17] introduced the affine arclength measure to obtain optimal $L^{p_{2}} \rightarrow L^{q_{2}}$ estimates for $(t, p(t))$, where $p(t)$ satisfies some technical assumptions. (See also [7].) Let $\gamma(t)=$ $\left(P_{1}(t), \ldots, P_{d}(t)\right)$ for arbitrary polynomials $P_{i}(t), 1 \leq i \leq d$. When $d=2$, Oberlin [29] showed optimal $L^{p_{2}} \rightarrow L^{q_{2}}$ boundedness of $\mathcal{B}$, where the constant depends only on the maximum degree of the polynomials. Dendrinos, Laghi and Wright [13] obtained the uniform boundedness of $\mathcal{B}$ in some Lorentz spaces when $d=3$, which was an extension of the case $\gamma(t)=\left(t, P_{1}(t), P_{2}(t)\right)$ established by Oberlin [29]. For the general dimension, see Stovall [34]. For more details on the history related to general classes of curves, we refer to $[13,34]$ and the references therein.

Recently, Dendrinos and Stovall [15] proved the restricted weak type estimates for certain curves (not necessarily simple) with low regularity under monotonicity and concavity assumptions on the torsions. Their result is a generalization of Oberlin's work [31]. In [15], it is also considered strong type $\left(p_{d}, q_{d}\right)$ bounds for perturbation of monomial curves. By suitably ordering certain parameters, they efficiently avoid the band structure argument.

We wish to point out here that the affine arclength measure was first used in the study of the Fourier restriction estimates for various classes of degenerate curves to allow the possibility of uniform estimates by mitigating the degeneracy of the torsion of the curve. (See [3-5,14,16,17,19,20,23,35] for the case of degenerate real curves, and see [1,30] for complex curves.)

In the study of the Fourier restriction estimates for space curves, properties of the mapping $\left(t_{1}, \ldots, t_{d}\right) \mapsto \sum_{i=1}^{d} \gamma\left(t_{i}\right)$, such as finite generic multiplicity and a lower bound for the Jacobian, are required in order to perform a change of variables.

The usual treatment of the affine arclength measure involved a lower bound of a certain Jacobian in terms of the geometric mean of the torsion. Here, following [1,5], we will exploit a stronger estimate involving the arithmetic mean (or, equivalently, the maximum) of the torsion rather than their geometric mean. (See Lemma 1.) The stronger geometric inequalities allow us to obtain a slightly stronger estimate and to simplify some arguments such as those in [13,34]. (See Proposition 1 and the proof of Lemma 12.)

The main ingredients of this paper are an extension of the band structure argument and geometric inequalities for the Jacobian. To handle the higher-dimensional case $d>3$ in Theorem 1, we extend the band structure argument under the lack of order structure of complex variables. To get around this obstacle, we consider balls which are separated from each other. Then we examine the number of variables contained in the balls. See Sect. 3 for details. Then the proof of Theorem 1 is completed in Sect. 4.

To prove Theorem 2, the band structure argument is not essential. In this case, the main result is a Jacobian estimate for finite type complex curves. (See Lemma 7.) Since we get the 
weaker geometric mean rather than the arithmetic mean in Lemma 7, we give a brief proof of Theorem 2 in Sect. 5. We wish to point out that the modified band structure argument in Sect. 3 is motivated by the proof of Lemma 14.

Throughout this paper, we write $A \lesssim B$, or $B \lesssim A$, if there exists a constant $C>0$ such that $A \leq C B$, or $B \leq C A$, respectively. If we write $A \sim B$, it means both $A \lesssim B$ and $B \lesssim A$. Also $A \ll B$ denotes $A \leq C^{-1} B$ for a large constant $C$.

\section{Lower bounds for the Jacobian}

\subsection{Simple type curves}

First we briefly review the lower bound estimates for the Jacobian in [1]. Let $\Gamma(z)=$ $\left(z, z^{2}, \ldots, z^{d-1}, \phi_{d}(z)\right)$ where $\phi_{d}(z)=z^{N}, N \geq d$ for $d>3$ and $\phi_{3}(z)$ is an arbitrary complex polynomial of degree $N \geq 3$. In [1], they obtained a uniform lower bound of the Jacobian of the mapping $\left(z_{1}, \ldots, z_{d}\right) \mapsto \sum_{j=1}^{d} \Gamma\left(z_{j}\right)$ as follows.

Lemma 1 (Lemmas 3.3 and 4.2 in [1]) Let $\Gamma(z)=\left(z, z^{2}, \ldots, z^{d-1}, \phi_{d}(z)\right)$ as above. Set $J_{\mathbb{C}}\left(z_{1}, \ldots, z_{d}\right)=\operatorname{det}\left(\Gamma^{\prime}\left(z_{1}\right), \ldots, \Gamma^{\prime}\left(z_{d}\right)\right)$ where $z_{j} \in \mathbb{C}, 1 \leq j \leq d$. There exists a positive integer $M=M(N)$ and a collection of convex open sets $B_{1}, \ldots, B_{M}$, which are pairwise disjoint, such that $\mathbb{C}=\bigcup_{\ell=1}^{M} B_{\ell}$ ignoring null sets. Moreover, there exists a constant $c(N)>$ 0 such that for $1 \leq \ell \leq M$,

$$
\left|J_{\mathbb{C}}\left(z_{1}, \ldots, z_{d}\right)\right| \geq c(N) V\left(z_{1}, \ldots, z_{d}\right) \max _{1 \leq j \leq d}\left|\phi_{d}^{(d)}\left(z_{j}\right)\right|,
$$

whenever $z_{j} \in B_{\ell}$ for $1 \leq j \leq d$. Here $V\left(z_{1}, \ldots, z_{d}\right)=\prod_{1 \leq i<j \leq d}\left|z_{j}-z_{i}\right|$ is the Vandermonde determinant.

By the proof of Lemma 3.3 in [1], for $d>3$ the set $B_{\ell}$ 's are sectors $\Delta_{\ell}$ with vertex at the origin. For $d=3$, the proof of Lemma 4.2 in [1] shows that $B_{\ell}$ 's are certain convex subsets of $\Delta_{\ell}$ after additional decomposition of $\mathbb{C}$ depending on zeros of $\phi_{3}(z)$. In addition, on each $B_{\ell}$, there exists a constant $L_{\ell}$ and $k_{\ell}$ such that

$$
\left|\phi_{3}^{\prime \prime \prime}(z)\right| \sim L_{\ell}|z|^{k_{\ell}} .
$$

Note that $2 \phi_{3}^{\prime \prime \prime}(z)$ is the torsion of $\Gamma(z)=\left(z, z^{2}, \phi_{3}(z)\right)$.

\subsection{Monomial curves with integer orders}

To deal with finite type curves in $\mathbb{C}^{3}$, we begin with the simplest case of finite type curves. Let $\Gamma(z)=\left(z^{a_{1}}, z^{a_{2}}, z^{a_{3}}\right)$ for distinct integers $a_{i}$ 's satisfying $1 \leq a_{1}<a_{2}<a_{3}$. Then

$$
\left|\tau_{\Gamma}(z)\right|:=\left|\operatorname{det}\left(\Gamma^{\prime}(z) \Gamma^{\prime \prime}(z) \Gamma^{\prime \prime \prime}(z)\right)\right|=C\left(a_{1}, a_{2}, a_{3}\right)|z|^{a_{1}+a_{2}+a_{3}-6} .
$$

for the positive constant $C\left(a_{1}, a_{2}, a_{3}\right)=a_{1} a_{2} a_{3}\left(a_{2}-a_{1}\right)\left(a_{3}-a_{1}\right)\left(a_{3}-a_{2}\right)$.

Lemma 2 Let $\Gamma(z)$ be given as above. Then $\mathbb{C}$ can be decomposed into finitely many sectors $\Delta_{\ell}\left(\ell\right.$ depends on $\left.a_{i}\right)$ with vertex at the origin, and there exists a constant $C$, independent of $a_{i}$, such that for $z_{i} \in \Delta_{\ell}$,

$$
\left|J_{\mathbb{C}}\left(z_{1}, z_{2}, z_{3}\right)\right| \geq C \prod_{i=1}^{3}\left|\tau_{\Gamma}\left(z_{i}\right)\right|^{\frac{1}{3}} \prod_{1 \leq i<j \leq 3}\left|z_{i}-z_{j}\right| .
$$


Recall the definition of monic polynomials in $z_{1}, z_{2}, z_{3}$ in [1].

Definition 1 Let $N$ be a nonnegative integer and let $z_{1}, z_{2}, z_{3}$ be complex numbers. We define a homogeneous monic polynomial $P_{N}$ of degree $N$ by

$$
P_{N}\left(z_{1}, z_{2}, z_{3}\right)=\sum_{\substack{\alpha_{1}+\alpha_{2}+\alpha_{3}=N \\ \alpha_{1}, \alpha_{2}, \alpha_{3} \geq 0}} z_{1}^{\alpha_{1}} z_{2}^{\alpha_{2}} z_{3}^{\alpha_{3}} .
$$

To prove Theorem 2, we make use of the following properties of $P_{N}$ :

\section{Lemma 3 Let $N \geq 1$. Then}

(i)

$$
P_{0}\left(z_{1}, z_{2}, z_{3}\right)=1 \text {, }
$$

(ii)

$$
\begin{aligned}
P_{N}\left(z_{1}, z_{2}, z_{3}\right) & =z_{1}^{N}+z_{1}^{N-1} P_{1}\left(z_{2}, z_{3}\right)+\cdots+z_{1} P_{N-1}\left(z_{2}, z_{3}\right)+P_{N}\left(z_{2}, z_{3}\right) \\
& =z_{1} P_{N-1}\left(z_{1}, z_{2}, z_{3}\right)+P_{N}\left(z_{2}, z_{3}\right),
\end{aligned}
$$

$$
\begin{aligned}
& P_{N}\left(z_{1}, z_{3}\right)-P_{N}\left(z_{1}, z_{2}\right) \\
& =\left(z_{1}^{N}+z_{1}^{N-1} z_{3}+\cdots+z_{1} z_{3}^{N-1}+z_{3}^{N}\right)-\left(z_{1}^{N}+z_{1}^{N-1} z_{2}+\cdots+z_{1} z_{2}^{N-1}+z_{2}^{N}\right) \\
& =\left(z_{3}-z_{2}\right)\left(z_{1}^{N-1}+z_{1}^{N-2}\left(z_{3}+z_{2}\right)+\cdots+z_{1} P_{N-2}\left(z_{3}, z_{2}\right)+P_{N-1}\left(z_{3}, z_{2}\right)\right) \\
& =\left(z_{3}-z_{2}\right) P_{N-1}\left(z_{1}, z_{2}, z_{3}\right),
\end{aligned}
$$

$$
P_{N+K-1}\left(z_{1}, z_{2}\right)=z_{1}^{K} P_{N-1}\left(z_{1}, z_{2}\right)+z_{2}^{N} P_{K-1}\left(z_{1}, z_{2}\right)
$$

$$
P_{N}\left(z_{2} z_{3}, z_{3} z_{1}\right)=z_{3}^{N} P_{N}\left(z_{1}, z_{2}\right) .
$$

Lemma 4 For any integer $m \geq 1$,

$$
P_{m}\left(z_{1}, z_{2}\right) P_{m}\left(z_{2}, z_{3}\right)-z_{2}^{m+1} P_{m-1}\left(z_{1}, z_{2}, z_{3}\right)=P_{m}\left(z_{1} z_{2}, z_{2} z_{3}, z_{3} z_{1}\right) .
$$

Moreover,

$$
\begin{aligned}
& P_{m-1}\left(z_{1}, z_{2}\right) P_{m-1}\left(z_{1}, z_{2}, z_{3}\right)-P_{m-2}\left(z_{1}, z_{2}, z_{3}\right) P_{m}\left(z_{1}, z_{2}\right) \\
& \quad=P_{m-1}\left(z_{1} z_{2}, z_{2} z_{3}, z_{3} z_{1}\right) .
\end{aligned}
$$

Proof First we show (5). The case $m=1$ is clear. Now we assume that (5) holds for $m=k$. By (ii), (iv) and (v) in Lemma 3, we have

$$
\begin{aligned}
& P_{k+1}\left(z_{1}, z_{2}\right) P_{k+1}\left(z_{2}, z_{3}\right)-z_{2}^{k+2} P_{k}\left(z_{1}, z_{2}, z_{3}\right) \\
&= P_{k+1}\left(z_{1}, z_{2}\right)\left(z_{3}^{k+1}+z_{2} P_{k}\left(z_{2}, z_{3}\right)\right)-z_{2}^{k+2}\left(z_{2} P_{k-1}\left(z_{1}, z_{2}, z_{3}\right)+P_{k}\left(z_{1}, z_{3}\right)\right) \\
&= P_{k+1}\left(z_{2} z_{3}, z_{3} z_{1}\right) \\
&+z_{2}[\underbrace{P_{k+1}\left(z_{1}, z_{2}\right) P_{k}\left(z_{2}, z_{3}\right)-z_{2}^{k+2} P_{k-1}\left(z_{1}, z_{2}, z_{3}\right)-z_{2} P_{k}\left(z_{1} z_{2}, z_{2} z_{3}\right)}_{=: I}]
\end{aligned}
$$


Since $P_{k+1}\left(z_{1}, z_{2}\right)=z_{2} P_{k}\left(z_{1}, z_{2}\right)+z_{1}^{k+1}$,

$$
\begin{aligned}
I= & \left(z_{2} P_{k}\left(z_{1}, z_{2}\right)+z_{1}^{k+1}\right) P_{k}\left(z_{2}, z_{3}\right)-z_{2}^{k+2} P_{k-1}\left(z_{1}, z_{2}, z_{3}\right)-z_{2} P_{k}\left(z_{1} z_{2}, z_{2} z_{3}\right) \\
= & z_{2}\left(P_{k}\left(z_{1}, z_{2}\right) P_{k}\left(z_{2}, z_{3}\right)-z_{2}^{k+1} P_{k-1}\left(z_{1}, z_{2}, z_{3}\right)\right) \\
& +z_{1} P_{k}\left(z_{1} z_{2}, z_{3} z_{1}\right)-z_{2} P_{k}\left(z_{1} z_{2}, z_{2} z_{3}\right) \\
= & z_{2} P_{k}\left(z_{1} z_{2}, z_{2} z_{3}, z_{3} z_{1}\right)-z_{2} P_{k}\left(z_{1} z_{2}, z_{2} z_{3}\right)+z_{1} P_{k}\left(z_{1} z_{2}, z_{3} z_{1}\right) \\
= & z_{2}\left(z_{3} z_{1} P_{k-1}\left(z_{1} z_{2}, z_{2} z_{3}, z_{3} z_{1}\right)+P_{k}\left(z_{1} z_{2}, z_{2} z_{3}\right)\right) \\
& -z_{2} P_{k}\left(z_{1} z_{2}, z_{2} z_{3}\right)+z_{1} P_{k}\left(z_{1} z_{2}, z_{3} z_{1}\right) \\
= & z_{1} z_{2} z_{3} P_{k-1}\left(z_{1} z_{2}, z_{2} z_{3}, z_{3} z_{1}\right)+z_{1} P_{k}\left(z_{1} z_{2}, z_{3} z_{1}\right) \\
= & z_{1} P_{k}\left(z_{1} z_{2}, z_{2} z_{3}, z_{3} z_{1}\right) .
\end{aligned}
$$

Here the third equality follows from the induction assumption. Then we obtain

$$
\begin{aligned}
P_{k+1} & \left(z_{1}, z_{2}\right) P_{k+1}\left(z_{2}, z_{3}\right)-z_{2}^{k+2} P_{k}\left(z_{1}, z_{2}, z_{3}\right) \\
\quad & =P_{k+1}\left(z_{2} z_{3}, z_{3} z_{1}\right)+z_{2} z_{1} P_{k}\left(z_{1} z_{2}, z_{2} z_{3}, z_{3} z_{1}\right) \\
& =P_{k+1}\left(z_{2} z_{3}, z_{3} z_{1}\right)+z_{1} z_{2} P_{k}\left(z_{1} z_{2}, z_{2} z_{3}, z_{3} z_{1}\right) \\
& =P_{k+1}\left(z_{1} z_{2}, z_{2} z_{3}, z_{3} z_{1}\right),
\end{aligned}
$$

which implies (5).

By (ii), (iv) in Lemma 3, LHS of (6) can be written as

$$
\begin{aligned}
P_{m-1} & \left(z_{1}, z_{2}\right)\left(z_{1} P_{m-2}\left(z_{1}, z_{2}, z_{3}\right)+P_{m-1}\left(z_{2}, z_{3}\right)\right) \\
& \quad-P_{m-2}\left(z_{1}, z_{2}, z_{3}\right)\left(z_{1} P_{m-1}\left(z_{1}, z_{2}\right)+z_{2}^{m}\right) \\
& =P_{m-1}\left(z_{1}, z_{2}\right) P_{m-1}\left(z_{2}, z_{3}\right)-z_{2}^{m} P_{m-2}\left(z_{1}, z_{2}, z_{3}\right) .
\end{aligned}
$$

Then (6) is valid by (5).

The following lemma is a generalization of (6).

Lemma 5 Let $k \geq 0$ be an integer. Then

$$
\begin{aligned}
& P_{m-1}\left(z_{1}, z_{2}\right) P_{m+k-1}\left(z_{1}, z_{2}, z_{3}\right)-P_{m-2}\left(z_{1}, z_{2}, z_{3}\right) P_{m+k}\left(z_{1}, z_{2}\right) \\
& \quad=P_{k}\left(z_{1}, z_{2}\right) z_{1} z_{2} P_{m-2}\left(z_{1} z_{2}, z_{2} z_{3}, z_{3} z_{1}\right)+P_{k}\left(z_{1}, z_{2}, z_{3}\right) P_{m-1}\left(z_{2} z_{3}, z_{3} z_{1}\right)
\end{aligned}
$$

Proof We use mathematical induction on $k$. By (6) and (ii) in Lemma 3, (7) holds for $k=0$. Let us assume that (7) holds for $k=\ell-1$.

Using Lemma 3, we get

$$
\begin{aligned}
P_{m-1} & \left(z_{1}, z_{2}\right) P_{m+\ell-1}\left(z_{1}, z_{2}, z_{3}\right)-P_{m-2}\left(z_{1}, z_{2}, z_{3}\right) P_{m+\ell}\left(z_{1}, z_{2}\right) \\
= & P_{m-1}\left(z_{1}, z_{2}\right)\left(z_{1} P_{m+\ell-2}\left(z_{1}, z_{2}, z_{3}\right)+P_{m+\ell-1}\left(z_{2}, z_{3}\right)\right) \\
& -P_{m-2}\left(z_{1}, z_{2}, z_{3}\right)\left(z_{1} P_{m+\ell-1}\left(z_{1}, z_{2}\right)+z_{2}^{m+\ell}\right) \\
= & z_{1}\left(P_{m-1}\left(z_{1}, z_{2}\right) P_{m+\ell-2}\left(z_{1}, z_{2}, z_{3}\right)-P_{m-2}\left(z_{1}, z_{2}, z_{3}\right) P_{m+\ell-1}\left(z_{1}, z_{2}\right)\right) \\
& +P_{m-1}\left(z_{1}, z_{2}\right) P_{m+\ell-1}\left(z_{2}, z_{3}\right)-z_{2}^{m+\ell} P_{m-2}\left(z_{1}, z_{2}, z_{3}\right) \\
= & z_{1}\left(P_{m-1}\left(z_{1}, z_{2}\right) P_{m+\ell-2}\left(z_{1}, z_{2}, z_{3}\right)-P_{m-2}\left(z_{1}, z_{2}, z_{3}\right) P_{m+\ell-1}\left(z_{1}, z_{2}\right)\right) \\
& +P_{m-1}\left(z_{1}, z_{2}\right)\left(z_{2}^{\ell} P_{m-1}\left(z_{2}, z_{3}\right)+z_{3}^{m} P_{\ell-1}\left(z_{2}, z_{3}\right)\right)-z_{2}^{m+\ell} P_{m-2}\left(z_{1}, z_{2}, z_{3}\right) \\
= & {\left[z_{1}\left(P_{m-1}\left(z_{1}, z_{2}\right) P_{m+\ell-2}\left(z_{1}, z_{2}, z_{3}\right)-P_{m-2}\left(z_{1}, z_{2}, z_{3}\right) P_{m+\ell-1}\left(z_{1}, z_{2}\right)\right)\right] } \\
& +\left[z_{2}^{\ell}\left(P_{m-1}\left(z_{1}, z_{2}\right) P_{m-1}\left(z_{2}, z_{3}\right)-z_{2}^{m} P_{m-2}\left(z_{1}, z_{2}, z_{3}\right)\right)\right.
\end{aligned}
$$




$$
\begin{aligned}
& \left.+z_{3}^{m} P_{m-1}\left(z_{1}, z_{2}\right) P_{\ell-1}\left(z_{2}, z_{3}\right)\right] \\
= & :[I]+[I I] .
\end{aligned}
$$

By the induction assumption,

$$
\begin{aligned}
I= & z_{1}\left(P_{\ell-1}\left(z_{1}, z_{2}\right) z_{1} z_{2} P_{m-2}\left(z_{1} z_{2}, z_{2} z_{3}, z_{3} z_{1}\right)\right. \\
& \left.+P_{\ell-1}\left(z_{1}, z_{2}, z_{3}\right) P_{m-1}\left(z_{2} z_{3}, z_{3} z_{1}\right)\right) .
\end{aligned}
$$

By (5) and (ii), (v) in Lemma 3, we also get

$$
\begin{aligned}
I I= & z_{2}^{\ell} P_{m-1}\left(z_{1} z_{2}, z_{2} z_{3}, z_{3} z_{1}\right)+z_{3} P_{m-1}\left(z_{2} z_{3}, z_{3} z_{1}\right) P_{\ell-1}\left(z_{2}, z_{3}\right) \\
= & z_{2}^{\ell}\left(z_{1} z_{2} P_{m-2}\left(z_{1} z_{2}, z_{2} z_{3}, z_{3} z_{1}\right)+P_{m-1}\left(z_{2} z_{3}, z_{3} z_{1}\right)\right) \\
& +z_{3} P_{m-1}\left(z_{2} z_{3}, z_{3} z_{1}\right) P_{\ell-1}\left(z_{2}, z_{3}\right) .
\end{aligned}
$$

Hence it follows by (ii) in Lemma 3 that

$$
\begin{aligned}
I+I I= & \left(z_{1} P_{\ell-1}\left(z_{1}, z_{2}\right)+z_{2}^{\ell}\right) z_{1} z_{2} P_{m-2}\left(z_{1} z_{2}, z_{2} z_{3}, z_{3} z_{1}\right) \\
& +\left(z_{1} P_{\ell-1}\left(z_{1}, z_{2}, z_{3}\right)+z_{2}^{\ell}+z_{3} P_{\ell-1}\left(z_{2}, z_{3}\right)\right) P_{m-1}\left(z_{2} z_{3}, z_{3} z_{1}\right) \\
= & P_{\ell}\left(z_{1}, z_{2}\right) z_{1} z_{2} P_{m-2}\left(z_{1} z_{2}, z_{2} z_{3}, z_{3} z_{1}\right)+P_{\ell}\left(z_{1}, z_{2}, z_{3}\right) P_{m-1}\left(z_{2} z_{3}, z_{3} z_{1}\right) .
\end{aligned}
$$

This completes the proof.

Now we are ready to prove Theorem 2 .

Proof of Theorem 2 Let $m:=a_{2}-a_{1} \geq 1$ and $n:=a_{3}-a_{1} \geq 2$. Then we have

$$
\begin{aligned}
J_{\mathbb{C}} & =\left|\begin{array}{lll}
a_{1} z_{1}^{a_{1}-1} & a_{1} z_{2}^{a_{1}-1} & a_{1} z_{3}^{a_{1}-1} \\
a_{2} z_{1}^{a_{2}-1} & a_{2} z_{2}^{a_{2}-1} & a_{2} z_{3}^{a_{2}-1} \\
a_{3} z_{1}^{a_{3}-1} & a_{3} z_{2}^{a_{3}-1} & a_{3} z_{3}^{a_{3}-1}
\end{array}\right|=\prod_{i=1}^{3} a_{i} z_{i}^{a_{1}-1}\left|\begin{array}{ccc}
1 & 1 & 1 \\
z_{1}^{m} & z_{2}^{m} & z_{3}^{m} \\
z_{1}^{n} & z_{2}^{n} & z_{3}^{n}
\end{array}\right| \\
& =\prod_{i=1}^{3} a_{i} z_{i}^{a_{1}-1}\left|\begin{array}{cc}
z_{2}^{m}-z_{1}^{m} & z_{3}^{m}-z_{1}^{m} \\
z_{2}^{n}-z_{1}^{n} & z_{3}^{n}-z_{1}^{n}
\end{array}\right| \\
& =\prod_{i=1}^{3} a_{i} z_{i}^{a_{1}-1}\left(z_{2}-z_{1}\right)\left(z_{3}-z_{1}\right)\left|\begin{array}{ll}
P_{m-1}\left(z_{1}, z_{2}\right) & P_{m-1}\left(z_{1}, z_{3}\right) \\
P_{n-1}\left(z_{1}, z_{2}\right) & P_{n-1}\left(z_{1}, z_{3}\right)
\end{array}\right| \\
& =\prod_{i=1}^{3} a_{i} z_{i}^{a_{1}-1} \prod_{1 \leq i<j \leq 3}\left(z_{j}-z_{i}\right)\left|\begin{array}{ll}
P_{m-1}\left(z_{1}, z_{2}\right) & P_{m-2}\left(z_{1}, z_{2}, z_{3}\right) \\
P_{n-1}\left(z_{1}, z_{2}\right) & P_{n-2}\left(z_{1}, z_{2}, z_{3}\right) .
\end{array}\right|
\end{aligned}
$$

Let us set $k=n-m-1 \geq 0$. By (7), we see that

$$
\begin{aligned}
& \left|\begin{array}{ll}
P_{m-1}\left(z_{1}, z_{2}\right) & P_{m-2}\left(z_{1}, z_{2}, z_{3}\right) \\
P_{n-1}\left(z_{1}, z_{2}\right) & P_{n-2}\left(z_{1}, z_{2}, z_{3}\right)
\end{array}\right| \\
& \quad=P_{k}\left(z_{1}, z_{2}\right) z_{1} z_{2} P_{m-2}\left(z_{1} z_{2}, z_{2} z_{3}, z_{3} z_{1}\right)+P_{k}\left(z_{1}, z_{2}, z_{3}\right) P_{m-1}\left(z_{2} z_{3}, z_{3} z_{1}\right) \\
& \quad=: \mathcal{P}\left(z_{1}, z_{2}, z_{3}\right) .
\end{aligned}
$$

Hence if we assume that

$$
\left|\mathcal{P}\left(z_{1}, z_{2}, z_{3}\right)\right| \gtrsim \prod_{1 \leq i<j \leq 3}\left(a_{j}-a_{i}\right) \prod_{i=1}^{3}\left|z_{i}\right|^{\frac{2 m+k-2}{3}},
$$


then

$$
\begin{aligned}
\left|J_{\mathbb{C}}\right| & \gtrsim C\left(a_{1}, a_{2}, a_{3}\right) \prod_{i=1}^{3}\left|z_{i}\right|^{a_{1}-1-\frac{2 m+k-2}{3}} \prod_{1 \leq i<j \leq 3}\left|z_{j}-z_{i}\right| \\
& =C\left(a_{1}, a_{2}, a_{3}\right) \prod_{i=1}^{3}\left|z_{i}\right|^{\frac{a_{1}+a_{2}+a_{3}-6}{3}} \prod_{1 \leq i<j \leq 3}\left|z_{j}-z_{i}\right|
\end{aligned}
$$

which implies (4) since $\left|\tau_{\Gamma}(z)\right|=C\left(a_{1}, a_{2}, a_{3}\right)|z|^{a_{1}+a_{2}+a_{3}-6}$.

Now we show (8). Let $x_{i}$ and $y_{i}$ be the real and imaginary parts of $z_{i}$, respectively. By rotation, we may assume that $z_{i} \in \Delta=\left\{r e^{i \theta}: 0<r<1,0<\theta<\delta\right\}$ and $\delta$ will be chosen later. LHS of (8) is bounded below by the absolute value of its real part. Moreover

$$
\left|\operatorname{Re}\left(\mathcal{P}\left(z_{1}, z_{2}, z_{3}\right)\right)\right| \geq\left|\mathcal{P}\left(x_{1}, x_{2}, x_{3}\right)\right|-\left|\mathcal{E}\left(x_{i}, y_{i}\right)\right| .
$$

The remainder term $\mathcal{E}\left(x_{i}, y_{i}\right)$ is the sum of similar terms to $\mathcal{P}\left(x_{1}, x_{2}, x_{3}\right)$ in which at least one of $x_{i}$ is replaced by $C\left(a_{i}\right) y_{i}$ for a constant $C\left(a_{i}\right)$. Since $y_{i} \lesssim \delta x_{i}$ for $z_{i} \in \Delta$, it follows that $\left|\mathcal{E}\left(x_{i}, y_{i}\right)\right| \leq \frac{1}{2}\left|\mathcal{P}\left(x_{1}, x_{2}, x_{3}\right)\right|$ by choosing sufficiently small $\delta$ depending on $a_{i}$. So it suffices to show that $\mathcal{P}\left(x_{1}, x_{2}, x_{3}\right)$ is bounded below by RHS of $(8)$. ( $\mathcal{P}\left(x_{1}, x_{2}, x_{3}\right)$ is positive whenever $z_{i} \in \Delta$.)

We have

$$
\mathcal{P}(\mathbf{x}):=\mathcal{P}\left(x_{1}, x_{2}, x_{3}\right)=\operatorname{det}\left(\begin{array}{ccc}
1 & 1 & 1 \\
x_{1}^{m} & x_{2}^{m} & x_{3}^{m} \\
x_{1}^{n} & x_{2}^{n} & x_{3}^{n}
\end{array}\right) / \prod_{1 \leq i<j \leq 3}\left(x_{j}-x_{i}\right) .
$$

By [36, pp. 200-201] (see also [9]), $\mathcal{P}(\mathbf{x})$ is a homogeneous symmetric polynomial with nonnegative coefficients. Also its degree is $k+2 m-2$, and the number of terms is given by $N=m n(n-m) / 2$.

Let us set

$$
\mathcal{P}(\mathbf{x})=\sum_{\mathbf{e}=k+2 m-2} C_{\mathbf{e}} x_{1}^{e_{1}} x_{2}^{e_{2}} x_{3}^{e_{3}},
$$

for $\mathbf{e}=\sum e_{i}$ and $C_{\mathbf{e}} \geq 0$. By symmetry, it follows that

$$
\begin{aligned}
3 \mathcal{P}(\mathbf{x}) & =\sum_{\mathbf{e}=k+2 m-2} C_{\mathbf{e}}\left(x_{1}^{e_{1}} x_{2}^{e_{2}} x_{3}^{e_{3}}+x_{1}^{e_{2}} x_{2}^{e_{3}} x_{3}^{e_{1}}+x_{1}^{e_{3}} x_{2}^{e_{1}} x_{3}^{e_{2}}\right) \\
& \geq 3 N\left(x_{1} x_{2} x_{3}\right)^{(k+2 m-2) / 3} .
\end{aligned}
$$

Hence we get (8) since $x_{i} \sim\left|z_{i}\right|$ on $\Delta$ with a sufficiently small $\delta$ and $N=m n(n-m) / 2=$ $\left(a_{3}-a_{2}\right)\left(a_{3}-a_{1}\right)\left(a_{3}-a_{2}\right) / 2$.

\subsection{Bounds for perturbed monomial curves}

Recall that the class $\mathcal{G}^{\mathbf{a}}(\epsilon)$ of perturbed monomial curves given by

$$
\mathcal{G}^{\mathbf{a}}(\epsilon)=\left\{\Gamma(z)=\left(z^{a_{1}} \psi_{1}(z), z^{a_{2}} \psi_{2}(z), \ldots, z^{a_{d}} \psi_{d}(z)\right):\left\|\psi_{i}-1\right\|_{C^{a_{d}+1}(2 D)} \leq \epsilon\right\},
$$

where $a_{i}$ 's are integers satisfying $1 \leq a_{1}<a_{2}<\cdots<a_{d}$ and $\psi_{j}$ 's are complex-valued functions. 
In this section, we obtain Jacobian and torsion bounds for $\Gamma \in \mathcal{G}^{\mathbf{a}}(\epsilon)$. If $\epsilon$ is small enough, then the Jacobian and torsion bounds can be controlled by those of the monomial curve $\left(z^{a_{1}}, \ldots, z^{a_{d}}\right)$. By modifying the argument in [23] (see also [14]), we obtain the following.

Lemma 6 Let $\Gamma \in \mathcal{G}^{\mathbf{a}}(\epsilon)$. If $\epsilon>0$ is sufficiently small, then

$$
\left|\tau_{\Gamma}(z)\right| \sim|z|^{\sum_{i=1}^{d} a_{i}-d(d+1) / 2}
$$

holds. The implicit constant is independent of $\Gamma$.

Proof We define a function $\Psi_{i, j}(z)$ of the sum of derivatives of $\psi_{i}$ 's by

$$
\Psi_{i, j}(z)=\sum_{k=0}^{j-1} a_{i}\left(a_{i}-1\right) \cdots\left(a_{i}-(j-k-1)\right)\left(\begin{array}{l}
j \\
k
\end{array}\right) z^{k} \psi_{i}^{(k)}(z)+z^{j} \psi_{i}^{(j)}(z) .
$$

So $\frac{\mathrm{d}^{j}}{\mathrm{~d} z^{j}}\left(z^{a_{i}} \psi_{i}(z)\right)=z^{a_{i}-j} \Psi_{i, j}(z)$. Let us define constants $\Psi_{i, j}^{\circ}, 1 \leq i, j \leq d$, by

$$
\Psi_{i, j}^{\circ}=\prod_{k=0}^{j-1}\left(a_{i}-k\right) .
$$

Since $|z|<1, j \leq d<a_{d}$, and $\Gamma \in \mathcal{G}^{\mathbf{a}}(\epsilon)$, we see that

$$
\begin{aligned}
\left|\Psi_{i, j}(z)-\Psi_{i, j}^{\circ}\right| & \leq \prod_{k=0}^{j-1}\left(a_{i}-k\right)\left|\psi_{i}(z)-1\right|+\sum_{k=1}^{j-1} C_{\mathbf{a}}|z|^{k}\left|\psi_{i}^{(k)}(z)\right|+|z|^{j}\left|\psi_{i}^{(j)}(z)\right| \\
& \lesssim_{\mathbf{a}}\left\|\psi_{i}-1\right\|_{C^{a_{d}+1}(D)} \lesssim_{\mathbf{a}} \epsilon .
\end{aligned}
$$

Here we denote by $C_{\mathbf{a}}$ a constant depending on $\mathbf{a}$ and $\lesssim_{\mathbf{a}}$ means the implicit constant depends on $\mathbf{a}$.

Since $\Gamma^{(j)}(z)=\left(z^{a_{1}-j} \Psi_{1, j}(z), \ldots, z^{a_{d}-j} \Psi_{d, j}(z)\right)$ for $1 \leq j \leq d$, we have

$$
\left|\tau_{\Gamma}(z)\right|=|z|^{\sum_{i=1}^{d} a_{i}-d(d+1) / 2}\left|\operatorname{det}\left(\Psi_{i, j}(z)\right)\right| .
$$

By multilinearity of determinant and (9), we obtain $\left|\operatorname{det}\left(\Psi_{i, j}(z)\right)\right| \sim_{\mathbf{a}, d}\left|\operatorname{det}\left(\Psi_{i, j}^{\circ}\right)\right|$ for a sufficiently small $\epsilon$. Here $\left(\Psi_{i, j}^{\circ}\right)_{1 \leq i, j \leq d}$ is a constant matrix and

$$
\begin{aligned}
\operatorname{det}\left(\Psi_{i, j}^{\circ}\right) & =\operatorname{det}\left(\begin{array}{cccc}
a_{1} & a_{1}\left(a_{1}-1\right) & \cdots & \prod_{k=0}^{d-1}\left(a_{1}-k\right) \\
\vdots & \vdots & \vdots & \vdots \\
a_{d} & a_{d}\left(a_{d}-1\right) & \cdots & \prod_{k=0}^{d-1}\left(a_{d}-k\right)
\end{array}\right) \\
& =\prod_{i=1}^{d} a_{i} \prod_{1 \leq i<j \leq d}\left(a_{j}-a_{i}\right) .
\end{aligned}
$$

Since $a_{i}$ 's are distinct integers and $a_{i} \geq i$, we have $\operatorname{det}\left(\Psi_{i, j}^{\circ}\right) \neq 0$. So,

$$
\left|\tau_{\Gamma}(z)\right|=|z|^{\sum_{i=1}^{d} a_{i}-d(d+1) / 2}\left|\operatorname{det}\left(\Psi_{i, j}(z)\right)\right| \sim_{\mathbf{a}, d}|z|^{\sum_{i=1}^{d} a_{i}-d(d+1) / 2}
$$

This completes the proof.

For $d=3$, we obtain the following Jacobian estimate. 
Lemma 7 Let $\Gamma \in \mathcal{G}^{\mathbf{a}}(\epsilon)$. If $\epsilon$ is sufficiently small, there exist a constant $C=C(\mathbf{a}, \epsilon)$, $a$ number $N=N(\mathbf{a}, \epsilon)$ and a collection of sectors $\Delta_{\ell}$ with vertex at the origin such that $D=\cup_{0 \leq \ell \leq N} \Delta_{\ell}$ ignoring a measure zero set, and

$$
\left|J_{\mathbb{C}}\left(z_{1}, z_{2}, z_{3}\right)\right| \geq C \prod_{i=1}^{3}\left|\tau_{\Gamma}\left(z_{i}\right)\right|^{\frac{1}{3}} \prod_{1 \leq i<j \leq 3}\left|z_{i}-z_{j}\right|
$$

provided $z_{i} \in \Delta_{\ell}$.

Proof Using the notation $\Psi_{i, j}$ as in the proof of Lemma 6, we have

$$
J_{\mathbb{C}}\left(z_{1}, z_{2}, z_{3}\right)=\operatorname{det}\left(a_{i} z_{j}^{a_{i}-1} \Psi_{i, 1}\left(z_{j}\right)\right) .
$$

Let $\Psi_{1}=\Psi_{1,1}$ and $\Psi_{2}=\Psi_{1,1} \Psi_{2,2}-\Psi_{1,2} \Psi_{2,1}$ and $\Psi_{3}=\operatorname{det}\left(\Psi_{i, j}\right)$. Since $\left|\Psi_{i, j}\right| \gtrsim \Psi_{i, j}^{\circ}-\epsilon$ by (9), $\Psi_{i}$ 's are nonvanishing if $\epsilon$ is sufficiently small.

Using the argument due to Dendrinos (see [12,16]), the Jacobian can be written as

$$
J_{\mathbb{C}}\left(z_{1}, z_{2}, z_{3}\right)=\prod_{j=1}^{3} a_{j} z_{j}^{a_{1}-1} \Psi_{1}\left(z_{j}\right) \int_{z_{1}}^{z_{2}} \int_{z_{2}}^{z_{3}} \int_{u}^{v} F(u, v, w) \mathrm{d} w \mathrm{~d} v \mathrm{~d} u,
$$

where

$$
F(u, v, w)=(u v)^{a_{2}-a_{1}-1} w^{a_{3}-a_{2}-1} \frac{\Psi_{2}(u)}{\Psi_{1}(u)^{2}} \frac{\Psi_{2}(v)}{\Psi_{1}(v)^{2}} \frac{\Psi_{1}(w) \Psi_{3}(w)}{\Psi_{2}(w)^{2}} .
$$

Let $\Delta_{\ell}=\left\{r e^{i \theta} \in D: r>0, \ell \delta<\theta<(\ell+1) \delta\right\}$ for a small $\delta$ to be chosen later. Then we can decompose $D$, except for a measure zero set, into $O\left(\delta^{-1}\right)$ sectors $\Delta_{\ell}$. By rotation, we may assume that $z_{i}$ 's are contained in $\Delta_{0}$.

By change of variables $u=z_{1}+\left(z_{2}-z_{1}\right) t_{1}, v=z_{2}+\left(z_{3}-z_{2}\right) t_{2}$, and $w=u+(v-u) t_{3}$, it follows that

$$
\begin{aligned}
J_{\mathbb{C}}= & \prod_{j=1}^{3} z_{j}^{a_{1}-1} \Psi_{1}\left(z_{j}\right)\left(z_{2}-z_{1}\right)\left(z_{3}-z_{2}\right) \\
& \times \int_{0}^{1} \int_{0}^{1} \int_{0}^{1} F\left(u\left(t_{1}\right), v\left(t_{2}\right), w\left(t_{3}\right)\right)\left(v\left(t_{2}\right)-u\left(t_{1}\right)\right) \mathrm{d} t_{3} \mathrm{~d} t_{2} \mathrm{~d} t_{1} .
\end{aligned}
$$

Since $u$ is also contained in $\Delta_{0}, 0<\operatorname{Im}(u) \lesssim \delta \operatorname{Re}(u)$. Let $m:=a_{2}-a_{1}-1$ and $n:=a_{3}-a_{2}-1$. If $\delta=\delta(m)$ is sufficiently small, we have $\operatorname{Re}\left(u^{m}\right) \sim_{\delta}(\operatorname{Re} u)^{m} \sim_{\delta}|u|^{m}$. Moreover, there exists $\delta=\delta(m, n)$ such that $|u|^{m}|v|^{m}|w|^{n} \sim_{\delta} \operatorname{Re}\left(u^{m}\right) \operatorname{Re}\left(v^{m}\right) \operatorname{Re}\left(w^{n}\right) \sim_{\delta}$ $\operatorname{Re}\left(u^{m} v^{m} w^{n}\right)$.

Recalling the proof of Lemma 6, we see that there exists a constant $C$ depending on a such that $\left|\operatorname{det}\left(\Psi_{i, j}(z)\right)-\operatorname{det}\left(\Psi_{i, j}^{\circ}\right)\right| \leq C \epsilon$. So $\Psi_{k}$ 's for $k=1,2,3$ are so close to $\Psi_{k}^{\circ}:=\prod_{i=1}^{k} a_{i} \prod_{1 \leq i<j \leq k}\left(a_{j}-a_{i}\right) \in \mathbb{R}$ that $\left|\Psi_{k}-\Psi_{k}^{\circ}\right| \leq C_{k} \epsilon$ for a constant $C_{k}$. We set $\epsilon^{\prime}=\max _{1 \leq k \leq 3} C_{k} \epsilon$. Then $\left|\operatorname{Im} \Psi_{k}\right| \lesssim \frac{\epsilon^{\prime}}{\Psi_{k}^{\circ}+\epsilon^{\prime}} \operatorname{Re} \Psi_{k}$, so $\left|\Psi_{k}\right| \sim \operatorname{Re}\left(\Psi_{k}\right)$. It follows that

$$
\operatorname{Re}\left(\frac{\Psi_{2}(u)}{\Psi_{1}(u)^{2}}\right) \sim \frac{\operatorname{Re}\left(\Psi_{2}(u)\right) \operatorname{Re}\left(\overline{\Psi_{1}}(u)^{2}\right)}{\left|\Psi_{1}(u)\right|^{4}} \sim \frac{\left|\Psi_{2}(u)\right|\left|\overline{\Psi_{1}}(u)\right|^{2}}{\left|\Psi_{1}(u)\right|^{4}}=\frac{\left|\Psi_{2}(u)\right|}{\left|\Psi_{1}(u)\right|^{2}},
$$

where implicit constants depend on $\epsilon^{\prime}$. Similarly, we can see that $\operatorname{Re}\left(F^{\prime}(u, v, w)\right):=\operatorname{Re}\left(\frac{\Psi_{2}(u)}{\Psi_{1}(u)^{2}} \frac{\Psi_{2}(v)}{\Psi_{1}(v)^{2}} \frac{\Psi_{1}(w) \Psi_{3}(w)}{\Psi_{2}(w)^{2}}\right) \sim\left|\frac{\Psi_{2}(u)}{\Psi_{1}(u)^{2}} \frac{\Psi_{2}(v)}{\Psi_{1}(v)^{2}} \frac{\Psi_{1}(w) \Psi_{3}(w)}{\Psi_{2}(w)^{2}}\right|$ 
and $\left|\operatorname{Im}\left(F^{\prime}\right)\right| \lesssim C_{\epsilon^{\prime}} \operatorname{Re}\left(F^{\prime}\right)$. In addition there exists a constant $A$ such that $A^{-1} \Psi^{\circ} \leq\left|F^{\prime}\right| \leq$ $A \Psi^{\circ}$ for $\Psi^{\circ}=\Psi_{3}^{\circ} /\left(\Psi_{1}^{\circ}\right)^{3}$ if $\epsilon$ is sufficiently small.

Let $F(u, v, w)=\xi+i \zeta$. There exists a constant $c=c\left(\delta, \epsilon^{\prime}\right)$ such that $0<|\zeta|<c \xi$, and

$$
\xi=\operatorname{Re}\left(u^{m} v^{m} w^{n}\right) \operatorname{Re}\left(F^{\prime}\right)-\operatorname{Im}\left(u^{m} v^{m} w^{n}\right) \operatorname{Im}\left(F^{\prime}\right) \gtrsim_{c} \operatorname{Re}\left(u^{m} v^{m} w^{n}\right) \operatorname{Re}\left(F^{\prime}\right) \sim|F| .
$$

To obtain (11), we show that

$$
\begin{aligned}
& \left|\int_{[0,1]^{3}} F\left(u\left(t_{1}\right), v\left(t_{2}\right), w\left(t_{3}\right)\right)\left(v\left(t_{2}\right)-u\left(t_{1}\right)\right) \mathrm{d} t_{3} \mathrm{~d} t_{2} \mathrm{~d} t_{1}\right| \\
& \quad \gtrsim \int_{[0,1]^{3}}\left|u\left(t_{1}\right) v\left(t_{2}\right)\right|^{m}\left|w\left(t_{3}\right)\right|^{n}\left|v\left(t_{2}\right)-u\left(t_{1}\right)\right| \mathrm{d} t_{3} \mathrm{~d} t_{2} \mathrm{~d} t_{1} .
\end{aligned}
$$

We decompose each $\Delta_{\ell}$ into convex sets. For the convex hull $A_{k}$ of an annulus $\{k \delta / 2<$ $|z|<(k+1) \delta / 2\}$, we set $H_{\ell, k}=\Delta_{\ell} \cap\left(A_{k} \backslash A_{k+1}\right)$. Now we assume that $z_{1}, z_{2}, z_{3} \in H_{\ell, k}$. Let $\theta$ be the largest interior angle of the triangle $z_{1} z_{2} z_{3}$. Then $\pi / 3 \leq \theta \leq \pi$. We may assume that $\theta$ is the angle at $z_{2}$ and $\left|z_{3}-z_{2}\right| \geq\left|z_{2}-z_{1}\right|$. Using the affine invariance of (11), we can also assume that $z_{2}-z_{1}$ is a real number. In fact, via translation the triangle $z_{1} z_{2} z_{3}$ can be located in $\Delta_{\ell}$ such that $z_{2}-z_{1} \in \Delta_{\ell}$, i.e., the argument of $z_{2}-z_{1}$ is about $\ell \delta$. After rotating the sector $\Delta_{\ell}$, we see that the triangle $z_{1} z_{2} z_{3}$ is in the sector $\Delta_{0} \cup \Delta_{-1}$. We will choose a sufficiently small $\delta$ so that $|F| \sim \xi$ still holds for $u, v, w \in \Delta_{0} \cup \Delta_{-1}$.

Let $v\left(t_{2}\right)-u\left(t_{1}\right)=\alpha+i \beta$. Recall that $u, v$ are in the line segment $\left[z_{1}, z_{2}\right]$ and $\left[z_{2}, z_{3}\right]$, respectively.

Case $1 \pi / 3 \leq \theta<\pi / 2$. Let $t_{1}$ be fixed. Since $\beta$ is single signed, we may assume that $\beta>0$. It is enough to show that $\left|\int_{[0,1]^{3}}(\xi \beta+\zeta \alpha)\right|$ is bounded below by the right-hand side of (13) since $\operatorname{Im}\left(F\left(u\left(t_{1}\right), v\left(t_{2}\right), w\left(t_{3}\right)\right)\left(v\left(t_{2}\right)-u\left(t_{1}\right)\right)\right)=\xi \beta+\zeta \alpha$.

Let $t_{2}^{\circ}=t_{2}^{\circ}\left(t_{1}\right) \in[0,1]$ be the minimum of $t_{2}$ such that $\beta \geq|\alpha| / 2$ for $t_{2} \geq t_{2}^{\circ}$. Note that $t_{2}^{\circ} \leq 1 / 2$ by our assumptions. Let $L=L\left(t_{1}\right)$ be the distance between $u\left(t_{1}\right)$ and $\left[z_{2}, z_{3}\right]$. Then for all $t_{2} \leq 2 t_{2}^{\circ}$, we have $L \leq\left|v\left(t_{2}\right)-u\left(t_{1}\right)\right| \leq 2 L$.

Since $z_{i}$ 's are fixed, there exists a constant $M$ such that $M / 2 \leq\left|v\left(t_{2}\right)\right|^{m} \leq 2 M$ for any $t_{2}$. We have $|F| \sim M\left|u\left(t_{1}\right)\right|^{m}\left|w\left(t_{3}\right)\right|^{n}$ since $\left|F^{\prime}\right| \sim 1$ for a sufficiently small $\epsilon$. By the fact that $\left(1+c^{2}\right)^{-1 / 2}|F| \leq \xi \leq|F|$, it follows that

$$
\int_{0}^{t_{2}^{\circ}} \int_{0}^{1} \xi|v-u| \mathrm{d} t_{3} \mathrm{~d} t_{2} \lesssim L t_{2}^{\circ} M|u|^{m} \int_{0}^{1}|w|^{n} \mathrm{~d} t_{3} \lesssim C_{1} \int_{t_{2}^{\circ}}^{2 t_{2}^{\circ}} \int_{0}^{1} \xi|v-u| \mathrm{d} t_{3} \mathrm{~d} t_{2},
$$

where $C_{1}=\left(1+c^{2}\right)^{1 / 2}$. So,

$$
\begin{aligned}
\int_{t_{2}^{\circ}}^{1} \int_{0}^{1} \xi \beta \mathrm{d} t_{3} \mathrm{~d} t_{2} & \gtrsim \int_{2 t_{2}^{\circ}}^{1} \int_{0}^{1} \xi|v-u|+\int_{t_{2}^{\circ}}^{2 t_{2}^{\circ}} \int_{0}^{1} \xi|v-u| \\
& \gtrsim \int_{2 t_{2}^{\circ}}^{1} \int_{0}^{1} \xi|v-u|+\frac{1}{2} \int_{t_{2}^{\circ}}^{2 t_{2}^{\circ}} \int_{0}^{1} \xi|v-u|+\frac{1}{2 C_{1}} \int_{0}^{t_{2}^{\circ}} \int_{0}^{1} \xi|v-u| \\
& \geq \frac{1}{2 C_{1}} \int_{0}^{1} \int_{0}^{1} \xi|v-u| \mathrm{d} t_{3} \mathrm{~d} t_{2} .
\end{aligned}
$$

It follows that

$$
\int_{\beta \geq|\alpha| / 2} \xi \beta=\int_{0}^{1} \int_{t_{2}^{\circ}}^{1} \int_{0}^{1} \xi \beta \mathrm{d} t_{3} \mathrm{~d} t_{2} \mathrm{~d} t_{1} \geq \frac{1}{2 C_{1}} \int_{[0,1]^{3}} \xi|v-u| \mathrm{d} t_{3} \mathrm{~d} t_{2} \mathrm{~d} t_{1} .
$$


Therefore, we obtain

$$
\begin{aligned}
\left|\int_{[0,1]^{3}} \xi \beta+\zeta \alpha\right| & \geq\left|\int \xi \beta\right|-\int|\zeta \alpha| \geq \int_{\beta \geq|\alpha| / 2} \xi \beta-\int c \xi|v-u| \\
& \geq\left(\frac{1}{2 C_{1}}-c\right) \int|F||v-u| .
\end{aligned}
$$

By choosing sufficiently small $\delta$ and $\epsilon^{\prime}$ such that $c$ is small, we get $|F| \gtrsim \epsilon^{\prime}|u|^{m}|v|^{m}|w|^{n}$ and (13) follows.

Case $2 \pi / 2 \leq \theta \leq \pi$. In this case, we have $\alpha>0$, and we may assume that $\beta>0$ again. Since $\int|F||v-u| \leq \int C_{1} \xi(\alpha+\beta)$, we consider two cases.

If $\alpha \geq \beta / 2$, then $\int|F||v-u| \leq 3 C_{1} \int \xi \alpha$. So, LHS of (13) is bounded below by

$$
\begin{aligned}
& \left|\int_{[0,1]^{3}} \operatorname{Re}\left(F\left(u\left(t_{1}\right), v\left(t_{2}\right), w\left(t_{3}\right)\right)\left(v\left(t_{2}\right)-u\left(t_{1}\right)\right)\right)\right|=\left|\int \xi \alpha-\zeta \beta\right| \\
& \quad \geq \frac{1}{3 C_{1}} \int|F||v-u|-c \int \xi|v-u| \\
& \quad \geq\left(\frac{1}{3 C_{1}}-c\right) \int|F||v-u| .
\end{aligned}
$$

Then (13) holds by choosing sufficiently small $c$.

If $\alpha<\beta / 2$, then we have $\int|F||v-u| \leq 3 C_{1} / 2 \int \xi \beta$. It follows that $\left|\int \xi \beta+\zeta \alpha\right| \geq$ $\left(2 /\left(3 C_{1}\right)-c\right) \int|F||v-u|$. So we can get (13) again.

By (12) and (13), we obtain

$$
\begin{aligned}
\left|J_{\mathbb{C}}\left(z_{1}, z_{2}, z_{3}\right)\right| \gtrsim & \prod_{j=1}^{3}\left|z_{j}\right|^{a_{1}-1}\left|\Psi_{1}\left(z_{j}\right)\right|\left|z_{2}-z_{1}\right|\left|z_{3}-z_{2}\right| \\
& \times \int_{[0,1]^{3}}\left|u\left(t_{1}\right) v\left(t_{2}\right)\right|^{m}\left|w\left(t_{3}\right)\right|^{n}\left|v\left(t_{2}\right)-u\left(t_{1}\right)\right| \mathrm{d} t_{3} \mathrm{~d} t_{2} \mathrm{~d} t_{1} \\
\geq & \prod_{j=1}^{3}\left|\Psi_{1}\left(z_{j}\right)\right| \mid \prod_{j=1}^{3} z_{j}^{a_{1}-1}\left(z_{2}-z_{1}\right)\left(z_{3}-z_{2}\right) \\
& \times \int_{[0,1]^{3}} u\left(t_{1}\right)^{m} v\left(t_{2}\right)^{m} w\left(t_{3}\right)^{n}\left(v\left(t_{2}\right)-u\left(t_{1}\right)\right) \mathrm{d} t_{3} \mathrm{~d} t_{2} \mathrm{~d} t_{1} \mid \\
= & \prod_{j=1}^{3}\left|\Psi_{1}\left(z_{j}\right)\right|\left|\tilde{J}_{\mathbb{C}}\left(z_{1}, z_{2}, z_{3}\right)\right|,
\end{aligned}
$$

where $\widetilde{J}_{\mathbb{C}}\left(z_{1}, z_{2}, z_{3}\right)$ is the Jacobian of the mapping $\left(z_{1}, z_{2}, z_{3}\right) \mapsto \sum_{i=1}^{3} \widetilde{\Gamma}\left(z_{i}\right)$ for $\widetilde{\Gamma}(z)=$ $\left(z^{a_{1}}, z^{a_{2}}, z^{a_{3}}\right)$. Applying the proof of Proposition 2, there exists $\delta_{\circ}$ such that (4) also holds for $\widetilde{J}_{\mathbb{C}}\left(z_{1}, z_{2}, z_{3}\right)$ if each $z_{i}$ is contained in a narrow sector $\Delta$ with an angle $\delta_{\circ}$. Now we choose small $\delta$ and $\epsilon$ such that $\delta \leq \delta_{\circ}$ and $\xi \gtrsim|F|$. Then (11) holds whenever $z_{i} \in \Delta_{0}=\left\{r e^{i \theta} \in\right.$ $D: r>0,0<\theta<\delta\}$. 


\section{A restricted weak type estimate}

In this section, we consider the simple type curves in Theorem 1. By Lemma 1, we may restrict the domain $D$ of the convolution operator $\mathcal{A}$ to a convex open subset $B_{\ell}$. By rotation, we may assume that $B_{\ell}$ is a subset of the sector $\Delta_{0}=\left\{r e^{i \theta} \in D: r>0,0<\theta<\delta\right\}$. From now on, $\Delta$ denotes one of $B_{\ell} \cap \Delta_{0}$ so that Lemma 1 holds.

So it suffices to consider the convolution operator

$$
T f(x)=\int_{\Delta} f(x-\Gamma(z)) W_{\Gamma}(z) \mathrm{d} \mu(z) .
$$

By the proof of Lemma $1, W_{\Gamma}(z) \sim|z|^{\frac{4 K}{d(d+1)}}$ for $z \in \Delta$ and for an integer $K \geq 0$. Set $d \sigma(z)=|z|^{\frac{4 K}{d(d+1)}} \mathrm{d} \mu(z)$.

Let us define quantities $\alpha$ and $\beta$ by

$$
\alpha=\frac{\left\langle T \chi_{E}, \chi_{F}\right\rangle}{|F|} \text { and } \beta=\frac{\left\langle T^{*} \chi_{F}, \chi_{E}\right\rangle}{|E|},
$$

for measurable sets $E, F$. Here $T^{*}$ is the dual operator of $T$ given by $T^{*} f(x)=\int_{\Delta} f(x+$ $\Gamma(z)) \mathrm{d} \sigma(z)$. Since $\alpha|F|=\beta|E|$, the restricted weak type $\left(p_{d}, q_{d}\right)$ estimate

$$
\left\langle T \chi_{E}, \chi_{F}\right\rangle \lesssim|E|^{\frac{1}{p_{d}}}|F|^{\frac{1}{q_{d}^{\prime}}}
$$

is equivalent to

$$
|E| \gtrsim \alpha^{\frac{d(d+1)}{2}}\left(\frac{\beta}{\alpha}\right)^{d-1} \quad \text { or } \quad|F| \gtrsim \alpha^{\frac{d(d+1)}{2}}\left(\frac{\beta}{\alpha}\right)^{d} .
$$

To prove Theorem 1, we begin with the following which is one of refined versions of (15). The refined version for $F$ will be given in Sect. 4 .

Proposition 1 For $d \geq 3$, let $E_{1}, E_{2}, G \subset \mathbb{R}^{2 d}$ be measurable sets with finite measure. Suppose

$$
T \chi_{E_{1}}(x) \geq \alpha_{1} \text { and } T \chi_{E_{2}}(x) \geq \alpha_{2} \text { for all } x \in G,
$$

and $\alpha_{1} \leq \alpha_{2}$. Then

$$
\left|E_{2}\right| \gtrsim \alpha_{1}^{\frac{d(d+1)}{2}}\left(\frac{\beta}{\alpha_{1}}\right)^{d-1}\left(\frac{\alpha_{2}}{\alpha_{1}}\right)^{d},
$$

where $\beta=\alpha_{1}|G| /\left|E_{1}\right|$.

Remark 2 In view of Lemma 1, we may use the arithmetic mean of the torsion at $z_{1}, \ldots, z_{d}$ in place of the geometric mean to bound the Jacobian from below, which in turn enables us to obtain the slightly stronger estimate (17) than those in $[13,34]$. If we apply the geometric mean as in $[13,34]$, one can show that

$$
\left|E_{2}\right| \gtrsim \alpha_{1}^{\frac{d(d+1)}{2}}\left(\frac{\beta}{\alpha_{1}}\right)^{A_{d}}\left(\frac{\alpha_{2}}{\alpha_{1}}\right)^{\frac{d+1}{2}+\frac{d(d+1)}{4 K+2 d(d+1)}(d-1)},
$$

which is a weaker estimate because $d \geq \frac{d+1}{2}+\frac{d(d+1)}{4 K+2 d(d+1)}(d-1)$. 
We consider two cases $\beta \gtrsim \alpha_{1}$ and $\beta \ll \alpha_{1}$. While the former case can be treated by a straightforward method using Lemmas 8 and 9, the latter case requires refined estimate of Lemma 9 obtained by the modified band structure argument. (See Lemma 11.)

Lemma 8 Let $v=\frac{d(d+1)}{4 K+2 d(d+1)}$. Suppose that (16) holds and let $\gamma=\max \left\{\alpha_{1}, \beta\right\}$ for $\beta=$ $\alpha_{1}|G| /\left|E_{1}\right|$ as above. Then there exists a sequence of subsets $P_{1}, \ldots, P_{2 d}$ of $\Delta$ such that

$$
\sigma\left(P_{j}\right) \gtrsim \begin{cases}\beta, & \text { for odd } j, \\ \alpha_{1}, & \text { for even } j<2 d, \\ \alpha_{2}, & \text { for } j=2 d,\end{cases}
$$

where the implicit constants depend only on $d$. Also there exists a constant $c_{v}>0$ such that on $P_{j}$,

$$
\left|z_{j}\right| \geq \begin{cases}c_{\nu} \gamma^{\nu}, & \text { for } 1 \leq j \leq 2 d-1, \\ c_{\nu} \alpha_{2}^{\nu}, & \text { for } j=2 d .\end{cases}
$$

Proof The proof is a slight modification of previous works. (See $[10,13,22,33,34]$.) Let $B_{\gamma}$ be an open ball centered at the origin of radius $(2 \pi)^{-v} \gamma^{v}$ so that $\sigma\left(B_{\gamma}\right) \leq \gamma / 2$. We consider a truncated operator $\widetilde{T}$ instead of $T$, which is given by

$$
\widetilde{T} f(x)=\int_{\Delta \backslash B_{\gamma}} f(x-\Gamma(z)) \mathrm{d} \sigma(z) .
$$

Then we see that $\left\langle\widetilde{T} \chi_{E_{1}}, \chi_{G}\right\rangle \geq\left\langle T \chi_{E_{1}}, \chi_{G}\right\rangle-\sigma\left(B_{\gamma}\right)|G| \geq\left(\alpha_{1}-\gamma / 2\right)|G|$. If $\gamma=\alpha_{1}$, then $\left\langle\widetilde{T} \chi_{E_{1}}, \chi_{G}\right\rangle \geq \frac{1}{2} \alpha_{1}|G|=\frac{1}{2} \beta\left|E_{1}\right|$. If $\gamma=\beta$, we see that $\left\langle\widetilde{T} \chi_{E_{1}}, \chi_{G}\right\rangle=\left\langle\chi_{E_{1}}, \widetilde{T}^{*} \chi_{G}\right\rangle \geq$ $\left\langle\chi_{E_{1}}, T^{*} \chi_{G}\right\rangle-\sigma\left(B_{\gamma}\right)\left|E_{1}\right| \geq \frac{1}{2} \beta\left|E_{1}\right|$ since $\left\langle\chi_{E_{1}}, T^{*} \chi_{G}\right\rangle \geq \alpha_{1}|G|=\beta\left|E_{1}\right|$.

Therefore, we obtain

$$
\left\langle\widetilde{T} \chi_{E_{1}}, \chi_{G}\right\rangle \geq \frac{1}{2} \alpha_{1}|G|=\frac{1}{2} \beta\left|E_{1}\right|
$$

By abuse of notation, we will write $T$, instead of $\widetilde{T}$. By (20) there exists a subset $E_{1}^{1} \subset E_{1}$ given by $E_{1}^{1}=\left\{y \in E_{1}: T^{*} \chi_{G}(y) \geq \beta / 4\right\}$. Also we set $G^{1}=\left\{x \in G: T \chi_{E_{1}^{1}}(x) \geq \alpha_{1} / 8\right\}$, considering that $\left\langle T \chi_{E_{1}^{1}}, \chi_{G}\right\rangle=\left\langle T \chi_{E_{1}}, \chi_{G}\right\rangle-\left\langle\chi_{E_{1} \backslash E_{1}^{1}}, T^{*} \chi_{G}\right\rangle \geq \frac{1}{2} \beta\left|E_{1}\right|-\frac{1}{4} \beta\left|E_{1}\right|=$ $\frac{1}{4} \beta\left|E_{1}\right|=\frac{1}{4} \alpha_{1}|G|$.

Iterating this, we obtain sequences of subsets $E_{1}^{j}$ of $E_{1}$ and $G^{j}$ of $G$ such that

$$
\begin{aligned}
E_{1}^{0} & :=E_{1}, \quad G^{0}:=G \\
E_{1}^{j} & =\left\{y \in E_{1}^{j-1}: T^{*} \chi_{G^{j-1}}(y) \geq \beta / 2^{2 j}\right\}, \quad j=1, \ldots, d \\
G^{j} & =\left\{x \in G^{j-1}: T \chi_{E_{1}^{j}}(x) \geq \alpha_{1} / 2^{2 j+1}\right\}, \quad j=1, \ldots, d-1 .
\end{aligned}
$$

It is easy to verify that $E_{1}^{j}$ 's and $G^{j}$ 's are nonempty since

$$
\begin{aligned}
\left\langle T \chi_{E_{1}^{j+1}}, \chi_{G^{j+1}}\right\rangle & \geq\left\langle\chi_{E_{1}^{j}}, T^{*} \chi_{G^{j}}\right\rangle-\left\langle\chi_{E_{1}^{j} \backslash E_{1}^{j+1}}, T^{*} \chi_{G^{j}}\right\rangle-\left\langle T \chi_{E_{1}^{j+1}}, \chi_{G^{j} \backslash G^{j+1}}\right\rangle \\
& \geq 2^{-2 j-1} \alpha_{1}|G|-2^{-2 j-2} \alpha_{1}|G|-2^{-2 j-3} \alpha_{1}|G|=2^{-2 j-3} \beta\left|E_{1}\right|
\end{aligned}
$$


Now we define

$$
H_{k}\left(z_{1}, \ldots, z_{k}\right):=\sum_{j=1}^{k}(-1)^{j+1} \Gamma\left(z_{j}\right), \quad k \geq 1 .
$$

Fix any $y_{0} \in E_{1}^{d} \subset E_{1}$ and set $P_{1}=\left\{z_{1} \in \Delta \backslash B_{\gamma}: y_{0}+H_{1}\left(z_{1}\right) \in G^{d-1}\right\}$, then $\sigma\left(P_{1}\right)=$ $T^{*} \chi_{G^{d-1}}\left(y_{0}\right) \geq \beta / 2^{2 d}$. By the iterative process, we obtain that for $k=1,2,3, \ldots, d$, except for $P_{2 d}$,

$$
\begin{aligned}
P_{2 k-1} & =\left\{z_{2 k-1} \in \Delta \backslash B_{\gamma}: y_{0}+H_{2 k-1}\left(z_{1}, \ldots, z_{2 k-1}\right) \in G^{d-k}\right\}, \\
P_{2 k} & =\left\{z_{2 k} \in \Delta \backslash B_{\gamma}: y_{0}+H_{2 k}\left(z_{1}, \ldots, z_{2 k}\right) \in E_{1}^{d-k}\right\},
\end{aligned}
$$

provided $z_{j} \in P_{j}$. Finally we set $P_{2 d}=\left\{z_{2 d} \in \Delta \backslash B_{\alpha_{2}}: y_{0}+H_{2 d}\left(z_{1}, \ldots, z_{2 d}\right) \in E_{2}\right\}$ provided $z_{j} \in P_{j}$ for $j<2 d$.

Then it follows that for $k=2,3, \ldots, d$,

$$
\begin{aligned}
\sigma\left(P_{2 k-1}\right) & =T^{*} \chi_{G^{d-k}}\left(y_{0}+H_{2 k-2}\left(z_{1}, \ldots, z_{2 k-2}\right)\right) \geq \beta / 2^{2(d-k+1)} \\
\sigma\left(P_{2 k}\right) & =T \chi_{E_{1}^{d-k}}\left(y_{0}+H_{2 k-1}\left(z_{1}, \ldots, z_{2 k-1}\right)\right) \geq \alpha_{1} / 2^{2(d-k)+1} .
\end{aligned}
$$

In fact, we have

$$
\begin{aligned}
\sigma\left(P_{2 d}\right) & =\int_{\Delta \backslash B_{\alpha_{2}}} \chi_{E_{2}}\left(y_{0}+H_{2 d}\left(z_{1}, \ldots, z_{2 d}\right)\right) \mathrm{d} \sigma\left(z_{2 d}\right) \\
& \geq \int_{\Delta} \chi_{E_{2}}\left(y_{0}+H_{2 d}\left(z_{1}, \ldots, z_{2 d}\right)\right) \mathrm{d} \sigma\left(z_{2 d}\right)-\sigma\left(B_{\alpha_{2}}\right) \geq \frac{1}{2} \alpha_{2} .
\end{aligned}
$$

Hence we get a sequence of sets $P_{j}, 1 \leq j \leq 2 d$ satisfying (18) and (19). This completes the proof.

Lemma 9 Suppose that (18) and (19) hold. Then there exists a small constant $c>0$ and a sequence of sets $P_{j}^{\prime} \subset P_{j}, 1 \leq j \leq 2 d$ such that for $z_{j} \in P_{j}^{\prime}$,

(i) if $j<2 d$ is odd and $i<j$, then $\left|z_{j}-z_{i}\right| \geq c \beta^{\frac{1}{2}}\left|z_{i}\right|^{-\frac{2 K}{d(d+1)}}$,

(ii) if $j<2 d$ is even and $i<j$, then $\left|z_{j}-z_{i}\right| \geq c \alpha_{1}^{\frac{1}{2}}\left|z_{i}\right|^{-\frac{2 K}{d(d+1)}}$,

(iii) if $j=2 d$ and $i<2 d$, then

$$
\left|z_{2 d}-z_{i}\right| \geq \begin{cases}c \alpha_{2}^{\frac{1}{2}}\left|z_{2 d}\right|^{-\frac{2 K}{d(d+1)}} & \text { if }\left|z_{i}\right|<\frac{1}{2} c_{\nu} \alpha_{2}^{\nu} \\ c \alpha_{2}^{\frac{1}{2}}\left|z_{i}\right|^{-\frac{2 K}{d(d+1)}} & \text { if }\left|z_{i}\right| \geq \frac{1}{2} c_{\nu} \alpha_{2}^{\nu}\end{cases}
$$

Here $c_{v}$ is as in Lemma 8.

Proof We show (iii) first. Let us set

$$
B_{\beta}\left(z_{i}\right)=\left\{z \in \Delta:\left|z-z_{i}\right|<c_{0} \beta^{\frac{1}{2}}\left|z_{i}\right|^{-\frac{2 K}{d(d+1)}}\right\},
$$

where $c_{0}>0$ is a small constant to be chosen later. Recalling $v=d(d+1) /(4 K+2 d(d+1))$, it is easy to see that

$$
|z| \geq c_{\nu} \alpha^{v} \Longleftrightarrow|z| \geq c_{\nu}^{\frac{1}{2 v}} \alpha^{\frac{1}{2}}|z|^{-\frac{2 K}{d(d+1)}} .
$$

Case 1 If $\left|z_{i}\right|<\frac{1}{2} c_{\nu} \alpha_{2}^{\nu}$, then (19) and (22) give

$$
\left|z_{2 d}-z_{i}\right|>\frac{\left|z_{2 d}\right|}{2} \geq \frac{1}{2} c_{\nu}^{\frac{1}{2 v}} \alpha_{2}^{\frac{1}{2}}\left|z_{2 d}\right|^{-\frac{2 K}{d(d+1)}} .
$$


Case 2 Let $\left|z_{i}\right| \geq \frac{1}{2} c_{\nu} \alpha_{2}^{\nu}$. For $z \in B_{\alpha_{2}}\left(z_{i}\right)$, it follows from (22) that $\left|z-z_{i}\right| \leq$ $c_{0} \alpha_{2}^{1 / 2}\left|z_{i}\right|^{-2 K /(d(d+1))}<2 c_{0} c_{v}^{-1 / 2 v}\left|z_{i}\right|$, which implies $|z|<\left(1+2 c_{0} c_{v}^{-1 / 2 v}\right)\left|z_{i}\right|$.

Then

$$
\begin{aligned}
\sigma\left(B_{\alpha_{2}}\left(z_{i}\right)\right) & =\int_{B_{\alpha_{2}}\left(z_{i}\right)}|z|^{\frac{4 K}{d(d+1)}} \mathrm{d} \mu(z) \\
& <\left(1+2 c_{0} c_{\nu}^{-\frac{1}{2 v}}\right)^{\frac{4 K}{d(d+1)}}\left|z_{i}\right|^{\frac{4 K}{d(d+1)}} \times \pi c_{0}^{2} \alpha_{2}\left|z_{i}\right|^{-\frac{4 K}{d(d+1)}} \\
& =: c_{1} \alpha_{2}
\end{aligned}
$$

where $c_{1}=\pi c_{0}^{2}\left(1+2 c_{0} c_{v}^{-1 / 2 v}\right)^{\frac{4 K}{d(d+1)}}$. Choosing sufficiently small $c_{0}$, we have $\sigma\left(P_{2 d} \backslash\right.$ $\left.B_{\alpha_{2}}\left(z_{i}\right)\right)>\alpha_{2} / 2^{2 d+1}$. Note that Lemma 8 still holds for $P_{2 d}^{\prime}:=P_{2 d} \backslash B_{\alpha_{2}}\left(z_{i}\right)$ in place of $P_{2 d}$. Then $\left|z_{2 d}-z_{i}\right| \geq c_{0} \alpha_{2}^{\frac{1}{2}}\left|z_{j}\right|^{-\frac{2 K}{d(d+1)}}$ on $P_{2 d}^{\prime}$. Since Case 1 also holds on $P_{2 d}^{\prime} \subset P_{2 d}$, (iii) is valid.

We can obtain (i), (ii) similarly. In those cases, Case 2 only happens since $\left|z_{i}\right| \geq c_{\nu} \gamma^{\nu}$ for $1 \leq i \leq 2 d-1$.

Now we are ready to prove Proposition 1 when $\beta \gtrsim \alpha_{1}$. Let us set $\Phi_{2 d}=\left\{\left(z_{1}, z_{2}, \ldots, z_{2 d}\right)\right.$ : $z_{i} \in P_{i}^{\prime}$ where $\left.1 \leq i \leq 2 d\right\}$. Fix $z_{0}:=\left(z_{1}, z_{2}, \ldots, z_{d}\right) \in \Phi_{d}$ and set $\Phi:=\left\{z \in \mathbb{C}^{d}\right.$ : $\left.\left(z_{0}, z\right) \in \Phi_{2 d}\right\}$. If we set $\sigma^{d}$ is to be the $d$-fold product measure of $\sigma$, Lemma 8 gives $\sigma^{d}(\Phi) \gtrsim \alpha_{1}^{d / 2-1} \beta^{d / 2} \alpha_{2}$ when $d$ is even, and $\sigma^{d}(\Phi) \sim \alpha_{1}^{(d-1) / 2} \beta^{(d-1) / 2} \alpha_{2}$ when $d$ is odd. For $d \geq 2$, let

$$
A_{d}= \begin{cases}1+3+\cdots+(d-1) & \text { if } d \text { is even } \\ 2+4+\cdots+(d-1) & \text { if } d \text { is odd }\end{cases}
$$

Lemma 10 Let $d \geq 2$. Under the assumptions in Proposition 1,

$$
\left|E_{2}\right| \gtrsim \alpha_{1}^{\frac{d(d+1)}{2}}\left(\frac{\beta}{\alpha_{1}}\right)^{A_{d}}\left(\frac{\alpha_{2}}{\alpha_{1}}\right)^{d}
$$

holds. The implicit constant depends only on $d$.

Since $A_{d} \geq d-1$, this lemma implies (17) whenever $\beta \gtrsim \alpha_{1}$.

Proof We show the case when $d$ is even. The other case can be treated in a similar way. Let $H(z):=y_{0}+H_{2 d}\left(z_{0}, z\right)$. Since $H(\Phi) \subset E_{2}$ by the construction of $P_{2 d}$, it follows from Bezout's theorem and Lemma 1 that

$$
\begin{aligned}
\left|E_{2}\right| & \gtrsim \int_{\Phi}\left|J_{\mathbb{R}} H(z)\right| \mathrm{d} \mu(z)=\int_{\Phi}\left|J_{\mathbb{C}} H(z)\right|^{2} \mathrm{~d} \mu(z) \\
& \gtrsim \int_{\Phi} \max _{d+1 \leq i \leq 2 d}\left|z_{i}\right|^{2 K} \prod_{d+1 \leq i<j \leq 2 d}\left|z_{j}-z_{i}\right|^{2} \mathrm{~d} \mu(z),
\end{aligned}
$$

where $J_{\mathbb{R}} H$ is the real Jacobian of $H$ in $\mathbb{R}^{2 d}$. 
For $d+1 \leq i \leq 2 d-1$, we set $\varepsilon_{i}=0$ if $\left|z_{i}\right|<\frac{1}{2} c_{\nu} \alpha_{2}^{v}$, and $\varepsilon_{i}=1$ if $\left|z_{i}\right| \geq \frac{1}{2} c_{\nu} \alpha_{2}^{\nu}$. Using Lemma 9, we obtain

$$
\begin{aligned}
\left|E_{2}\right| \gtrsim & \alpha_{1}^{\frac{d(d-1)}{2}}\left(\frac{\beta}{\alpha_{1}}\right)^{2+4+\cdots+(d-2)}\left(\frac{\alpha_{2}}{\alpha_{1}}\right)^{d-1} \\
& \times \int_{\Phi} \max _{d+1 \leq i \leq 2 d}\left|z_{i}\right|^{2 K} \prod_{i=d+1}^{2 d-1}\left|z_{i}\right|^{\frac{-4 K}{d(d+1)}\left(2 d-i-1+\varepsilon_{i}\right)}\left|z_{2 d}\right|^{\frac{-4 K}{d(d+1)} \sum_{i=d+1}^{2 d-1}\left(1-\varepsilon_{i}\right)} \mathrm{d} \mu(z) .
\end{aligned}
$$

It is clear that $\max _{d+1 \leq i \leq 2 d}\left|z_{i}\right|^{2 K} \geq \prod_{i=d+1}^{2 d}\left|z_{i}\right|^{2 K_{i}}$ if $\sum_{i=d+1}^{2 d} K_{i}=K$. If we choose

$$
K_{i}=\frac{2 K}{d(d+1)}\left(1+(2 d-i-1)+\varepsilon_{i}\right) \text { and } K_{2 d}=\frac{2 K}{d(d+1)}\left(1+\sum_{i=d+1}^{2 d-1}\left(1-\varepsilon_{i}\right)\right),
$$

we obtain

$$
\begin{aligned}
\left|E_{2}\right| & \gtrsim \alpha_{1}^{\frac{d(d-1)}{2}}\left(\frac{\beta}{\alpha_{1}}\right)^{2+4+\cdots+(d-2)}\left(\frac{\alpha_{2}}{\alpha_{1}}\right)^{d-1} \int_{\Phi} \prod_{i=d+1}^{2 d}\left|z_{i}\right|^{\frac{4 K}{d(d+1)}} \mathrm{d} \mu(z) \\
& =\alpha_{1}^{\frac{d(d-1)}{2}}\left(\frac{\beta}{\alpha_{1}}\right)^{2+4+\cdots+(d-2)}\left(\frac{\alpha_{2}}{\alpha_{1}}\right)^{d-1} \sigma^{d}(\Phi) \\
& \gtrsim \alpha_{1}^{\frac{d(d+1)}{2}}\left(\frac{\beta}{\alpha_{1}}\right)^{A_{d}}\left(\frac{\alpha_{2}}{\alpha_{1}}\right)^{d},
\end{aligned}
$$

as desired.

\subsection{The band structure argument}

To handle the case $\beta \ll \alpha_{1}$, we modify the band structure argument due to Christ [10]. We also refer to the works by Stovall [34] and Gressman [22], which treat the degenerate cases.

Let $\mathcal{I}$ be a subset of $\{1, \ldots, 2 d\}$. We consider a partition of $\mathcal{I}$. Each element of the partition is called a band. Following the notion of [10], let us designate each index of a band as free, quasi-free, or bound by the rules below:

- In each band, the smallest index is a free index.

- If a band has only two indices, the bigger index is a quasi-free index.

- If a band has more than two indices, the other indices other than the free index are bound to the free index.

We say that a quasi-free index is quasi-bound to the free index in the band.

From now on we assume $z_{i} \in P_{i}^{\prime} \subset P_{i}, 1 \leq i \leq 2 d$, as in Lemmas 8 and 9.

Lemma 11 Let $\varepsilon>0$. There exist an integer $d \leq k<2 d$ and a partition of $\{2 d-k+1, \ldots, 2 d\}$. Also there exists a set $\omega \subset P_{2 d-k+1} \times \cdots \times P_{2 d}$ such that $\sigma^{k}(\omega) \gtrsim \alpha_{1}^{\lceil k / 2\rceil} \beta^{\lfloor k / 2\rfloor} \alpha_{2} / \alpha_{1}$. Finally, there exist parameters $\delta, \delta^{\prime}$ and a constant $c_{d, \varepsilon}>0$ satisfying $c_{d, \varepsilon} \leq \delta^{\prime}<\varepsilon \delta$ and a constant $c$ such that

(i) The number of free and quasi-free indices is $d$. In particular, each even index is free.

(ii) Unless $i$ and $j$ lie in the same band, $\left|z_{i}-z_{j}\right|>\delta \alpha_{1}^{\frac{1}{2}}\left|z_{i} z_{j}\right|^{-\frac{K}{d(d+1)}}$.

(iii) If $i$ is quasi-bound to $j, c \beta^{\frac{1}{2}}\left|z_{i} z_{j}\right|^{-\frac{K}{d(d+1)}}<\left|z_{i}-z_{j}\right| \leq \delta \alpha_{1}^{\frac{1}{2}}\left|z_{i} z_{j}\right|^{-\frac{K}{d(d+1)}}$. 
(iv) If $i$ is bound to $j, \delta^{\prime} \alpha_{1}^{\frac{1}{2}}\left|z_{i} z_{j}\right|^{-\frac{K}{d(d+1)}}>\left|z_{i}-z_{j}\right|$.

In the case for real variables, (ii) and (iii) are derived naturally by separation conditions like (23) and (24). Let $t_{i-1}<t_{i}<t_{i+1}$ be real variables for an example. If $i-1$ and $i$ satisfy (ii), so do $i-1$ and $i+1$ immediately. (To obtain (i) and (iv), we need an iteration process.)

Even though Lemma 11 is an analogue of one in the case for real variables, it does not seem likely that the original argument for the band structure can be applied directly to prove Lemma 11 under the lack of order structure of complex variables. Instead, we consider balls centered at $z_{j}$ for even $j$ which are mutually disjoint. Then we count odd indices which are bound to each even index.

Proof of Lemma 11 For even $j$ all the balls centered at $z_{j}$ with suitable radii can be mutually disjoint by the following observation.

Step 1: Separation conditions We can get simpler separation conditions than those in Lemma 9: there exists a constant $c$ such that if $i<j$,

$$
\begin{aligned}
& \left|z_{i}-z_{j}\right|>c \alpha_{1}^{\frac{1}{2}}\left|z_{i} z_{j}\right|^{-\frac{K}{d(d+1)}} \text { for even index } j \leq 2 d . \\
& \left|z_{i}-z_{j}\right|>c \beta^{\frac{1}{2}}\left|z_{i} z_{j}\right|^{-\frac{K}{d(d+1)}} \text { for odd index } j<2 d .
\end{aligned}
$$

These can be shown easily by triangular inequality. Let $j \leq 2 d$ be even and $i<j$. If $\left|z_{j}\right| \sim\left|z_{i}\right|$, then (23) is obvious. If $\left|z_{j}\right| \ll\left|z_{i}\right|$ or $\left|z_{j}\right| \gg\left|z_{i}\right|$, we have $\left|z_{i}-z_{j}\right| \gtrsim\left|z_{j}\right|=$ $\left|z_{j}\right|^{1+\frac{K}{d(d+1)}}\left|z_{i}\right|^{\frac{K}{d(d+1)}}\left|z_{i} z_{j}\right|^{-\frac{K}{d(d+1)}}$. Since $\left|z_{j}\right|,\left|z_{i}\right| \gtrsim \alpha_{1}^{v}$ by (19), it follows that

$$
\left|z_{i}-z_{j}\right| \gtrsim \alpha_{1}^{v\left(1+\frac{2 K}{d(d+1)}\right)}\left|z_{i} z_{j}\right|^{-\frac{K}{d(d+1)}}=\alpha_{1}^{\frac{1}{2}}\left|z_{i} z_{j}\right|^{-\frac{K}{d(d+1)}}
$$

Therefore, (23) holds for any even $j \leq 2 d$ and $i<j$. Also (24) can be obtained if we replace $\alpha_{1}$ by $\beta$.

Step 2: Constructing a partition of $\{1, \cdots, 2 d\}$. Let $\delta \leq c /(2 d)$ be a small constant to be chosen later. We define a ball centered at $z_{j}$ by

$$
B_{\delta, \alpha_{1}}\left(z_{j}\right)=\left\{z:\left|z-z_{j}\right| \leq \delta \alpha_{1}^{\frac{1}{2}}\left|z z_{j}\right|^{-\frac{K}{d(d+1)}}\right\} .
$$

So, $B_{\delta, \alpha_{1}}\left(z_{j}\right)$ for even $j$ are mutually disjoint. In general, $z_{i}$ for odd $i$ may be contained in either exactly one of $B_{\delta, \alpha_{1}}\left(z_{j}\right)$ satisfying $j<i$ or none of them.

For certain indices $j$, we will construct subsets $b(j) \subset\{1,2, \ldots, 2 d\}$ such that

- $j \in b(j)$,

- $j_{1} \in b(j)$ if $z_{j_{1}} \in B_{\delta, \alpha_{1}}\left(z_{j}\right)$,

- $j_{k+1} \in b(j)$ if $z_{j_{k+1}} \in B_{\delta, \alpha_{1}}\left(z_{j_{k}}\right)$ for some $j_{k} \in b(j)$.

If there is no $j_{1}$ such that $z_{j_{1}} \in B_{\delta, \alpha_{1}}\left(z_{j}\right)$, then we set $b(j)=\{j\}$. Note that if $b(j)$ contains more than two indices, then all indices other than $j$ are odd and bigger than $j$. Then we can observe that

$$
\left|z_{j_{k}}\right| \sim\left|z_{j_{l}}\right| \text { whenever } j_{k} \text { and } j_{l} \text { are in the same band. }
$$


Let $l>k$. Since $z_{j_{k+1}} \in B_{\delta, \alpha_{1}}\left(z_{j_{k}}\right)$ and $\left|z_{j}\right| \geq c_{\nu} \alpha_{1}^{v}$ for all $j$ by (19), it follows that

$$
\begin{aligned}
\left|z_{j_{k}}\right| & \geq\left|z_{j_{l}}\right|-\left(\left|z_{j_{k}}-z_{j_{k+1}}\right|+\left|z_{j_{k+1}}-z_{j_{k+2}}\right|+\cdots+\left|z_{j_{l-1}}-z_{j_{l}}\right|\right) \\
& \geq\left|z_{j_{l}}\right|-\mathrm{d} \delta \alpha_{1}^{\frac{1}{2}}\left(c_{\nu}^{2} \alpha_{1}^{2 v}\right)^{-\frac{K}{d(d+1)}} \\
& =\left|z_{j_{l}}\right|-\mathrm{d} \delta c_{\nu}^{-\frac{2 K}{d(d+1)}} \alpha_{1}^{v} .
\end{aligned}
$$

Thus we have $\left|z_{j_{l}}\right| \leq \mathrm{d} c_{v}^{-\frac{2 K}{d(d+1)}} \delta \alpha_{1}^{v}+\left|z_{j_{k}}\right| \leq\left(1+\mathrm{d} c_{\nu}^{-1 / 2 v} \delta\right)\left|z_{j_{k}}\right|$. If we exchange $z_{j_{k}}$ and $z_{j_{l}}$, then it also holds that $\left|z_{j_{k}}\right| \leq\left(1+\mathrm{d} c_{v}^{-1 / 2 v} \delta\right)\left|z_{j_{l}}\right|$. Hence for any $j_{k}, j_{l} \in b(j)$

$$
(1+\bar{c} \delta)^{-1}\left|z_{j_{k}}\right| \leq\left|z_{j_{l}}\right| \leq(1+\bar{c} \delta)\left|z_{j_{k}}\right|
$$

where $\bar{c}=\mathrm{d} c_{v}^{-1 / 2 v}$. Choosing sufficiently small $\delta$ we get (25).

Now we show how to construct $b(j)$ for each even $j$. By (23), there is no $i<2 d$ such that $z_{i} \in B_{\delta, \alpha_{1}}\left(z_{2 d}\right)$. So, $b(2 d)=\{2 d\}$.

From now on we fix $z_{1}, \ldots, z_{2 d}$. Let us consider $b(2 d-2)$. By (23) any $z_{i}$ for $i<j=$ $2 d-2$ cannot be in $B_{\delta, \alpha_{1}}\left(z_{2 d-2}\right)$. On the other hand, $z_{2 d-1}$ may be contained in $B_{\delta, \alpha_{1}}\left(z_{2 d-2}\right)$. So $b(2 d-2)=\{2 d-2,2 d-1\}$ or $\{2 d-2\}$.

Let $j_{1}$ be one of the odd indices contained in $b(j)$. If there exists an odd index $\ell$ such that $z_{\ell} \in B_{\delta, \alpha_{1}}\left(z_{j_{1}}\right)$, then we denote it by $j_{2}$ (of course, $j_{2} \in b(j)$ ). For $z_{j_{2}}$ we consider $B_{\delta, \alpha_{1}}\left(z_{j_{2}}\right)$ and repeat the process as above. Hence, each $b(j)$ consists of a unique even index $j$ and some odd indices greater than $j$. Also we have $B_{\delta, \alpha_{1}}\left(z_{j_{k}}\right) \subset B_{c / 2, \alpha_{1}}\left(z_{j}\right)$ for all $j_{k} \in b(j)$.

Now we consider indices which belong to none of the $b(j)$ for even $j$. In this case, we choose the smallest index among the remaining indices. By (23) this index must be 1 . In fact, for all even $j, B_{c / 2, \alpha_{1}}\left(z_{j}\right)$ and $B_{c / 2, \alpha_{1}}\left(z_{1}\right)$ are disjoint. Then we can construct $b(1)$ by considering $B_{\delta, \alpha_{1}}\left(z_{1}\right)$ as above.

If there are still remaining indices, we choose the smallest one and repeat the procedure. In this case, we can construct bands containing odd indices only.

By this procedure, we obtain the bands $b(j)$ 's which constitute a partition of the index set $\{1, \ldots, 2 d\}$. Here $j$ represents the free index in $b(j)$. Each free index is the smallest element in the band. (All even indices and 1 are free indices.) Each $b(j)$ has less than $d$ elements because of (23). (The case where $b(j)$ has $d$ elements can only occur when $b(1)$ contains all odd indices or $b(2)$ contains all odd indices except for 1.)

Now we show (ii) and (iii). Let us assume that $j^{\prime}$ and $k^{\prime}$ are in different bands $b(j)$ and $b(k)$, respectively. By the construction of $b(j)$ and $b(k)$, it follows that $z_{j^{\prime}} \notin B_{\delta, \alpha_{1}}\left(z_{k^{\prime}}\right)$ and $z_{k^{\prime}} \notin B_{\delta, \alpha_{1}}\left(z_{j^{\prime}}\right)$. This implies (ii) immediately.

If $j^{\prime}$ is quasi-bound to $j$, i.e., $b(j)=\left\{j, j^{\prime}\right\}$, then $j^{\prime}$ must be an odd number. Hence (iii) follows from the construction of $b(j)$ and (24).

Let $j_{k}$ be bound to $j$. By (25), we see that

$$
\begin{aligned}
\left|z_{j_{k}}-z_{j}\right| & \leq\left|z_{j_{k}}-z_{j_{k-1}}\right|+\cdots+\left|z_{j_{1}}-z_{j}\right| \\
& \leq \delta \alpha_{1}^{\frac{1}{2}}\left|z_{j_{k}} z_{j_{k-1}}\right|^{-\frac{K}{d(d+1)}}+\cdots+\delta \alpha_{1}^{\frac{1}{2}}\left|z_{j_{1}} z_{j}\right|^{-\frac{K}{d(d+1)}} \\
& \lesssim \mathrm{d} \delta \alpha_{1}^{\frac{1}{2}}\left|z_{j_{k}} z_{j}\right|^{-\frac{K}{d(d+1)}}
\end{aligned}
$$

In order to obtain (iv), we need an iteration procedure.

Step 3: Iteration procedure Let $\mathcal{P}:=P_{1} \times P_{2} \times \cdots \times P_{2 d}$ and $\delta^{\prime}=\epsilon \delta / 2$. If there exists a subset $\mathcal{P}_{1} \subset \mathcal{P}$ such that $\sigma^{d}\left(\mathcal{P}_{1}\right) \geq \sigma^{d}(\mathcal{P}) / 2$ and (iv) holds on $\mathcal{P}_{1}$, then we proceed to the next step. 
Otherwise, $\mathcal{P}_{1}$ occupies a relatively small portion of $\mathcal{P}$. Then on a relatively large subset $\mathcal{P}_{2} \subset \mathcal{P}$ there exist a band $b\left(j_{\circ}\right)$ and an index $i_{\circ} \in b\left(j_{\circ}\right)$ such that $i_{\circ}$ is bound to $j_{\circ}$ and $\left|z_{i_{\circ}}-z_{j_{0}}\right| \geq \delta^{\prime} \alpha_{1}^{\frac{1}{2}}\left|z_{i_{0}} z_{j_{0}}\right|^{-\frac{K}{d(d+1)}}$. Then we replace $\delta$ by $\delta^{\prime} / 2 d$ and $\delta^{\prime}$ by $\epsilon \delta^{\prime} / 4 d$. With these quantities, we repeat Step 2 on $\mathcal{P}_{2}$. Then all indices will be redefined once again. Then the number of free or quasi-free indices can increase, but (ii), (iii) still hold. If (iv) holds for $\epsilon \delta^{\prime} / 2 d$ in a subset which has large potion of $\mathcal{P}_{2}$, then we may proceed to the next step. Otherwise, we choose smaller $\delta, \delta^{\prime}$ and iterate the procedure again until we get

$$
\left|z_{j_{k}}-z_{j}\right|<\delta^{\prime} \alpha_{1}^{\frac{1}{2}}\left|z_{j_{k}} z_{j}\right|^{-\frac{K}{d(d+1)}}
$$

for each bound index $j_{k} \in b(j)$ on a subset $\widetilde{\mathcal{P}} \subset \mathcal{P}$. It is also valid that $\sigma^{d}(\widetilde{\mathcal{P}}) \gtrsim \sigma^{d}(\mathcal{P})$.

This procedure can be stopped in finite steps, so there exists a constant $c_{d, \epsilon}<\delta^{\prime}$.

Step 4: Discarding indices Adopting the notations in [10], we denote by $\mathcal{M}, \mathcal{N}$ the number of free and quasi-free indices, respectively. We have at least $d+1$ free indices, which are even indices and 1 , from the previous steps. Using a projection repeatedly, we will reduce the value $\mathcal{M}+\mathcal{N}$ to $d$, which yields (i). First, we discard the index 1 by fixing $z_{1} \in P_{1}$ and classify $\{2, \ldots, 2 d\}$ as free, quasi-free and bound indices. Then the number $\mathcal{M}+\mathcal{N}$ can decrease by one. Of course, it can be unchanged or increased by one when 1 was the free index of a band with two or three elements. After discarding indices appropriately, there exists an integer $k$ such that a band structure on $\{2 d-k+1, \ldots, 2 d\}$ satisfies $\mathcal{M}+\mathcal{N}=d$. Note that discarding an index does not affect properties (ii)-(iv).

Since $\widetilde{\mathcal{P}}=\widetilde{\mathcal{P}}_{2 d-k} \times \widetilde{\mathcal{P}}_{k}$, we can fix $z_{0} \in \widetilde{\mathcal{P}}_{2 d-k}$ so that there exists a subset $\omega \subset \widetilde{\mathcal{P}}_{k}$ such that $\sigma^{k}(\omega) \gtrsim \sigma^{k}\left(\widetilde{\mathcal{P}}_{k}\right) \gtrsim \alpha_{1}^{\lceil k / 2\rceil} \beta^{\lfloor k / 2\rfloor}\left(\alpha_{2} / \alpha_{1}\right)$ and (i)-(iv) holds on $\omega$.

\subsection{The slice argument}

Let $\Lambda=\left\{\lambda_{1}, \ldots, \lambda_{d}\right\} \subset\{2 d-k+1, \ldots, 2 d\}$ be the set of all indices which are free or quasi-free. Also we set $\tau=\left(\tau_{1}, \ldots, \tau_{d}\right) \in \mathbb{C}^{d}$ with $\tau_{i}=z_{\lambda_{i}}, \lambda_{i} \in \Lambda$. For $\lambda_{j}^{\prime} \in \Lambda^{\prime}:=$ $\{2 d-k+1, \ldots, 2 d\} \backslash \Lambda, 1 \leq j \leq k-d$, there is a free index $\lambda_{i} \in \Lambda$ to which $\lambda_{j}^{\prime}$ is bound. Let $s_{j}=\left(z_{\lambda_{j}^{\prime}}-z_{\lambda_{i}}\right) z_{\lambda_{i}}^{\frac{2 K}{d(d+1)}}$ and $s=\left(s_{1}, \ldots, s_{k-d}\right) \in \mathbb{C}^{k-d}$. It is clear that the map $z:=\left(z_{2 d-k+1}, \ldots, z_{2 d}\right) \mapsto(\tau, s)$ is a diffeomorphism, so its inverse $z(\tau, s)$ exists and is differentiable.

Recalling $H_{2 d}$ in $(21)$ we set $x_{0}+H_{2 d}\left(z_{1}, \ldots, z_{2 d-k}, z\right)=: H(z(\tau, s))$, then $H(\omega) \subset E_{2}$ for $\omega$ in Lemma 11.

For any $s \in \mathbb{C}^{k-d}$, we consider a slice $\omega_{s}=\{\tau: z(\tau, s) \in \omega\} \subset \mathbb{C}^{d}$. By the construction of $P_{2 d}$ in the proof of Lemma 8 (clearly $z_{2 d}$ is one of exponents of $\tau$ ), $H\left(\omega_{s}\right)$ is contained in $E_{2}$ for each $s$. By Bézout's theorem, for each $s \in \mathbb{C}^{k-d}$ there are finitely many preimages under the map $\tau \mapsto H(z(\tau, s))$. Thus we have

$$
\left|E_{2}\right| \gtrsim \int_{\omega_{s}}\left|\operatorname{det}\left(\frac{\partial H(z(\tau, s))}{\partial \tau}\right)(\tau, s)\right|^{2} \mathrm{~d} \mu(\tau) .
$$

The following lemma provides a lower bound for the integrand in (28). 
Lemma 12 For a given $\varepsilon>0$, there exist a band structure on $\{2 d-k+1, \ldots, 2 d\}$ and a set $\omega$ which satisfy Lemma 11. Then for all $(\tau, s) \in \omega$, and for some $C>0$,

$$
\left|\operatorname{det}\left(\frac{\partial H(z(\tau, s))}{\partial \tau}\right)\right|^{2} \geq C \alpha_{1}^{\frac{d(d-1)}{2}}\left(\frac{\beta}{\alpha_{1}}\right)^{\mathcal{N}}\left(\frac{\alpha_{2}}{\alpha_{1}}\right)^{d-1} \prod_{i=1}^{d}\left|\tau_{i}\right|^{\frac{4 K}{d(d+1)}}
$$

holds. Here $\mathcal{N}$ is the number of quasi-free indices associated with $\omega$. Also $\varepsilon$ and $C$ depend only on $d, \delta$ by Lemma 11.

We postpone its proof for a moment and prove Proposition 1.

\section{Proof of Proposition 1}

By integrating both sides of (28) and by (29), we have

$$
\begin{aligned}
& \int_{\{s: z(\tau, s) \in \omega\}}\left|E_{2}\right| \mathrm{d} \mu(s) \\
& \gtrsim \alpha^{\frac{d(d-1)}{2}}\left(\frac{\beta}{\alpha_{1}}\right)^{\mathcal{N}}\left(\frac{\alpha_{2}}{\alpha_{1}}\right)^{d-1} \int_{\{s: z(\tau, s) \in \omega\}} \int_{\omega_{s}} \prod_{i=1}^{d}\left|\tau_{i}\right|^{\frac{4 K}{d(d+1)}} \mathrm{d} \mu(\tau) \mathrm{d} \mu(s) .
\end{aligned}
$$

For any $s=\left(s_{1}, \ldots, s_{k-d}\right) \in \mathbb{C}^{k-d}$ satisfying $z(\tau, s) \in \omega$,

$$
\left|s_{j}\right|=\left|z_{\lambda_{j}^{\prime}}-z_{\lambda_{i}}\right|\left|z_{\lambda_{i}}\right|^{\frac{2 K}{d(d+1)}} \leq \delta^{\prime} \alpha_{1}^{\frac{1}{2}}\left|z_{\lambda_{j}^{\prime}} z_{\lambda_{i}}\right|^{-\frac{K}{d(d+1)}}\left|z_{\lambda_{i}}\right|^{\frac{2 K}{d(d+1)}} \lesssim \delta^{\prime} \alpha_{1}^{\frac{1}{2}}
$$

by (27) and (25). It follows that $\int_{\{s: z(\tau, s) \in \omega\}}\left|E_{2}\right| \mathrm{d} \mu(s) \lesssim \alpha_{1}^{k-d}\left|E_{2}\right|$.

Since $\left|\operatorname{det}\left(\frac{\partial(\tau, s)}{\partial z}\right)\right|^{2} \sim \prod_{j=1}^{k-d}\left|z_{\lambda_{j}^{\prime}}\right|^{\frac{4 K}{d(d+1)}}$ by (25), reversing the change of variables $(\tau, s) \mapsto z$ provides that

$$
\begin{aligned}
& \int_{\{s: z(\tau, s) \in \omega\}} \int_{\omega_{s}} \prod_{i=1}^{d}\left|\tau_{i}\right|^{\frac{4 K}{d(d+1)}} \mathrm{d} \mu(\tau) \mathrm{d} \mu(s) \\
& \sim \int_{\omega} \prod_{j=1}^{k-d}\left|z_{\lambda_{j}^{\prime}}\right|^{\frac{4 K}{d(d+1)}} \prod_{i=1}^{d}\left|z_{\lambda_{i}}\right|^{\frac{4 K}{d(d+1)}} \mathrm{d} \mu(z)=\sigma^{k}(\omega) .
\end{aligned}
$$

Hence we obtain

$$
\alpha_{1}^{k-d}\left|E_{2}\right| \gtrsim \alpha_{1}^{\frac{d(d-1)}{2}}\left(\frac{\beta}{\alpha_{1}}\right)^{\mathcal{N}}\left(\frac{\alpha_{2}}{\alpha_{1}}\right)^{d-1} \sigma^{k}(\omega),
$$

so

$$
\left|E_{2}\right| \gtrsim \alpha_{1}^{\frac{d(d+1)}{2}}\left(\frac{\beta}{\alpha_{1}}\right)^{\mathcal{N}+\lfloor k / 2\rfloor}\left(\frac{\alpha_{2}}{\alpha_{1}}\right)^{d} .
$$

This completes the proof since $\mathcal{N}+\lfloor k / 2\rfloor \leq d-1$ and $\beta \ll \alpha_{1}$.

\section{Proof of Lemma 12}

By definition of $\tau_{i}$ and $s_{j}$, we have

$$
\begin{aligned}
H(z(\tau, s))= & y_{0}+H_{2 d}\left(z_{1}, \ldots, z_{2 d-k}, z(\tau, s)\right) \\
= & y_{0}+H_{2 d-k}\left(z_{1}, \ldots, z_{2 d-k}\right) \\
& +\sum_{1 \leq i \leq d}\left(\theta_{i} \Gamma\left(\tau_{i}\right)+\sum_{j \Rightarrow i}(-1)^{\lambda_{j}^{\prime}+1}\left(\Gamma\left(s_{j} \tau_{i}^{-\frac{2 K}{d(d+1)}}+\tau_{i}\right)-\Gamma\left(\tau_{i}\right)\right)\right),
\end{aligned}
$$


where $j \Rightarrow i$ means $\lambda_{j}^{\prime} \in \Lambda^{\prime}$ is bound to $\lambda_{i} \in \Lambda$, and $\theta_{i}=(-1)^{\lambda_{i}+1}+\sum_{j \Rightarrow i}(-1)^{\lambda_{j}^{\prime}+1}$.

Note that $\theta_{i}$ cannot be 0 . In fact, for each $\lambda_{i}$, the number of indices which is bound to $\lambda_{i}$ is 0 or at least 2 . Also, all the indices bound to each $\lambda_{i}$ are odd. Thus, $\sum_{j \rightarrow i}(-1)^{\lambda_{j}^{\prime}+1}$ should be at least 2 (or 0 if there is no index bound to $i$ ).

For fixed $s$, each column of $\frac{\partial H(z(\tau, s))}{\partial \tau}$ is given by $\theta_{i} \Gamma^{\prime}\left(\tau_{i}\right)$ if there is no $j$ such that $j \Rightarrow i$, or given by

$$
\begin{aligned}
& \theta_{i} \Gamma^{\prime}\left(\tau_{i}\right)+\sum_{j \Rightarrow i}(-1)^{\lambda_{j}^{\prime}+1} \\
& \quad \times\left(\Gamma^{\prime}\left(s_{j} \tau_{i}^{-\frac{2 K}{d(d+1)}}+\tau_{i}\right)-\Gamma^{\prime}\left(\tau_{i}\right)-\frac{2 K}{d(d+1)}\left(\frac{s_{j}}{\tau_{i}}\right) \tau_{i}^{-\frac{2 K}{d(d+1)}} \Gamma^{\prime}\left(s_{j} \tau_{i}^{-\frac{2 K}{d(d+1)}}+\tau_{i}\right)\right) .
\end{aligned}
$$

Then by multilinearity, we have

$$
\operatorname{det}\left(\frac{\partial H(z(\tau, s))}{\partial \tau}\right)=C J_{d}(\tau)+\text { error terms, }
$$

where $C=\prod_{1 \leq i \leq d} \theta_{i}$, and $J_{d}(\tau)=\operatorname{det}\left(\Gamma\left(\tau_{1}\right), \ldots, \Gamma\left(\tau_{d}\right)\right)$ is the determinant of the complex Jacobian of the map $\left(\tau_{1}, \ldots, \tau_{d}\right) \mapsto \sum_{1 \leq i \leq d} \Gamma\left(\tau_{i}\right)$.

Our claim is that the error terms can be bounded by $O(\varepsilon) \times\left|J_{d}(\tau)\right|$. If it is proven, it follows that $\left|\operatorname{det}\left(\frac{\partial H(z(\tau, s))}{\partial \tau}\right)\right| \gtrsim\left|J_{d}(\tau)\right|$ by choosing sufficiently small $\varepsilon$. Recall that

$$
\left|J_{d}(\tau)\right| \gtrsim \max _{1 \leq i \leq d}\left|\tau_{i}\right|^{K} \prod_{1 \leq i<j \leq d}\left|\tau_{i}-\tau_{j}\right|
$$

in Lemma 1. We may assume that $\tau_{d}=z_{2 d}$. By the argument as in the proof of Lemma 10, we obtain (29).

Now we turn to the error terms, which are hybrids of the following terms:

$$
\begin{aligned}
I & :=\operatorname{det}\left(\Gamma^{\prime}\left(\tau_{1}\right), \ldots, \Gamma^{\prime}\left(\tau_{i-1}\right), \Gamma^{\prime}\left(u_{j(i)}+\tau_{i}\right)-\Gamma^{\prime}\left(\tau_{i}\right), \ldots, \Gamma^{\prime}\left(u_{j(d)}+\tau_{d}\right)-\Gamma^{\prime}\left(\tau_{d}\right)\right), \\
I I & :=\operatorname{det}\left(\Gamma^{\prime}\left(\tau_{1}\right), \ldots, \Gamma^{\prime}\left(\tau_{i-1}\right), \frac{u_{j(i)}}{\tau_{i}} \Gamma^{\prime}\left(u_{j(i)}+\tau_{i}\right), \ldots, \frac{u_{j(d)}}{\tau_{d}} \Gamma^{\prime}\left(u_{j(d)}+\tau_{d}\right)\right),
\end{aligned}
$$

where $u_{j(i)}=s_{j} \tau_{i}^{-\frac{2 K}{d(d+1)}}$ for some $j \Rightarrow i$.

To show $|I|,|I I| \leq O(\varepsilon)\left|J_{d}(\tau)\right|$, we make use of the fact that the bound indices are sufficiently close to the free index.

Let us denote by $z_{l^{\prime}}$ an index which is bound to $\tau_{l}$. Then $u_{j(l)}+\tau_{l}=z_{l^{\prime}}$ for $i \leq l \leq d$. By (iv) in Lemma 11, (25), and (22) we have

$$
\left|u_{j(l)}\right|=\left|z_{l^{\prime}}-\tau_{l}\right|<\varepsilon \delta \alpha_{1}^{\frac{1}{2}}\left|z_{l^{\prime}} \tau_{l}\right|^{-\frac{K}{d(d+1)}} \sim \varepsilon \delta \alpha_{1}^{\frac{1}{2}}\left|\tau_{l}\right|^{-\frac{2 K}{d(d+1)}} \lesssim \varepsilon \delta\left|\tau_{l}\right| .
$$

Let $z_{i^{\prime}}$ and $z_{l^{\prime}}$ are bound to $\tau_{i}$ and $\tau_{l}$, respectively. By triangular inequality and (30), we obtain

$$
\left|\tau_{i}-z_{l^{\prime}}\right| \sim\left|\tau_{i}-\tau_{l}\right| \text { and }\left|z_{i^{\prime}}-z_{l^{\prime}}\right| \sim\left|\tau_{i}-\tau_{l}\right| \text {. }
$$

The implicit constant depends only on $\varepsilon$.

First we show that $|I I| \leq O(\varepsilon)\left|J_{d}(\tau)\right|$. By (30), we have

$$
|I I| \lesssim(\varepsilon \delta)^{d-i+1}\left|\operatorname{det}\left(\Gamma^{\prime}\left(\tau_{1}\right), \ldots, \Gamma^{\prime}\left(\tau_{i-1}\right), \Gamma^{\prime}\left(z_{i^{\prime}}\right), \ldots, \Gamma^{\prime}\left(z_{d^{\prime}}\right)\right)\right| .
$$


By the proof of Lemma 7, we have that

$$
\left|\operatorname{det}\left(\Gamma^{\prime}\left(z_{1}\right) \cdots \Gamma^{\prime}\left(z_{d}\right)\right)\right| \sim \prod_{i=1}^{d}\left|\Psi_{i, 1}^{\circ}\right|\left|\operatorname{det}\left(h^{\prime}\left(z_{1}\right) \cdots h^{\prime}\left(z_{d}\right)\right)\right|,
$$

where $h(z)=\left(z, z^{2}, \ldots, z^{d-1}, z^{M}\right)$.

By the proof of Lemma 3.3 in [1],

$$
\operatorname{det}\left(h^{\prime}\left(z_{1}\right), \ldots, h^{\prime}\left(z_{d}\right)\right)=M(d-1) ! V\left(z_{1}, \ldots, z_{d}\right) P_{M-d}\left(z_{1}, \ldots, z_{d}\right),
$$

where $V\left(z_{1}, \ldots, z_{d}\right)=\prod_{1 \leq i<j \leq d}\left(z_{j}-z_{i}\right)$ is the complex Vandermonde determinant and $P_{m}$ is a homogeneous monic polynomial of degree $m$. (See Definition 1.)

By (31), it is easy to see that

$$
\left|V\left(\tau_{1}, \ldots, \tau_{i-1}, z_{i^{\prime}}, \ldots, z_{d^{\prime}}\right)\right| \lesssim\left|V\left(\tau_{1}, \ldots, \tau_{d}\right)\right| .
$$

Using (25), we also have

$$
\begin{aligned}
& \left|P_{M-d}\left(\tau_{1}, \ldots, \tau_{i-1}, z_{i^{\prime}}, \ldots, z_{d^{\prime}}\right)\right| \\
& \quad \leq \sum_{a_{1}+\cdots+a_{d}=N-d}\left|\tau_{1}\right|^{a_{1}} \cdots\left|\tau_{i-1}\right|^{a_{i-1}}\left|z_{i^{\prime}}\right|^{a_{i}} \cdots\left|z_{d^{\prime}}\right|^{a_{d}} \\
& \quad \sim \sum_{a_{1}+\cdots+a_{d}=N-d}\left|\tau_{1}\right|^{a_{1}} \cdots\left|\tau_{d}\right|^{a_{d}} \lesssim \max _{1 \leq i \leq d}\left|\tau_{i}\right|^{N-d} .
\end{aligned}
$$

Hence, we obtain

$$
|I I| \lesssim(\varepsilon \delta)^{d-i+1} \max _{1 \leq i \leq d}\left|\tau_{i}\right|^{N-d}\left|V\left(\tau_{1}, \ldots, \tau_{d}\right)\right| \lesssim(\varepsilon \delta)^{d-i+1}\left|J_{d}(\tau)\right| .
$$

Since we are assuming that $\Gamma \in \mathcal{G}^{\mathbf{a}}(\epsilon)$, we have

$$
\operatorname{det}\left(\Gamma^{\prime}\left(\tau_{1}\right) \cdots \Gamma^{\prime}\left(\tau_{d}\right)\right)=C \operatorname{det}\left(h^{\prime}\left(\tau_{1}\right) \cdots h^{\prime}\left(\tau_{d}\right)\right)+O(\epsilon)
$$

by the proof of Lemma 7. Then

$$
\left.I \sim \int_{\tau_{i}}^{u_{j(i)}+\tau_{i}} \cdots \int_{\tau_{d}}^{u_{j(d)}+\tau_{d}} \prod_{l=i}^{d} \frac{\partial}{\partial \tau_{l}}\right|_{\tau_{l}=\zeta_{l}} \operatorname{det}\left(h^{\prime}\left(\tau_{1}\right) \cdots h^{\prime}\left(\tau_{d}\right)\right) d \zeta_{d} \cdots d \zeta_{i}+O(\epsilon)
$$

By (32), we have

$$
\begin{aligned}
& \left.\prod_{l=i}^{d} \frac{\partial}{\partial \tau_{l}}\right|_{\tau_{l}=\zeta_{l}} \operatorname{det}\left(h^{\prime}\left(\tau_{1}\right) \cdots h^{\prime}\left(\tau_{d}\right)\right) \\
& \quad=\left.\left.M(d-1) ! \sum_{\left\{\mathfrak{I}_{1}, \Im_{2}\right\}} \prod_{l \in \mathfrak{I}_{1}} \frac{\partial}{\partial \tau_{l}}\right|_{\tau_{l}=\zeta_{l}} P_{M-d}\left(\tau_{1}, \ldots, \tau_{d}\right) \prod_{l \in \mathfrak{I}_{2}} \frac{\partial}{\partial \tau_{l}}\right|_{\tau_{l}=\zeta_{l}} V\left(\tau_{1}, \ldots, \tau_{d}\right),
\end{aligned}
$$

where $\left\{\mathfrak{I}_{1}, \mathfrak{I}_{2}\right\}$ is a partition of $\{i, i+1, \ldots, d\}$. 
Since $\zeta_{l}$ is on the line segment between $\tau_{l}$ and $u_{j(l)}+\tau_{l}$, we have $\left|\zeta_{l}\right| \sim\left|\tau_{l}\right|$ because of (30). Then we get

$$
\begin{aligned}
& \left|\prod_{l \in \mathfrak{I}_{1}} \frac{\partial}{\partial \tau_{l}}\right|_{\tau_{l}=\zeta_{l}} P_{M-d}\left(\tau_{1}, \ldots, \tau_{d}\right) \mid \\
& \quad=\left|\sum_{\substack{a_{1}+\ldots+a_{d}=M-d ; \\
a_{i}, \ldots, a_{d} \geq 1}}\left(\prod_{l \in \mathfrak{I}_{1}} a_{l}\right) \tau_{1}^{a_{1}} \ldots \tau_{i-1}^{a_{i-1}} \prod_{l \in \mathfrak{I}_{1}} \zeta_{l}^{a_{l}-1} \prod_{l \in \mathfrak{I}_{2}} \tau_{l}^{a_{l}}\right| \\
& \quad \lesssim \frac{\max _{1 \leq i \leq d}\left|\tau_{i}\right|^{M-d}}{\prod_{l \in \mathfrak{I}_{1}}\left|\tau_{l}\right|} .
\end{aligned}
$$

Since

$$
\frac{\partial}{\partial \tau_{l}} V\left(\tau_{1}, \ldots, \tau_{d}\right)=\sum_{\substack{1 \leq m \leq d ; \\ m \neq l}} \frac{V\left(\tau_{1}, \ldots, \tau_{d}\right)}{\tau_{l}-\tau_{m}},
$$

we see that

$$
\left|\prod_{l \in \mathfrak{I}_{2}} \frac{\partial}{\partial \tau_{l}} V\left(\tau_{1}, \ldots, \tau_{d}\right)\right| \leq \sum_{\substack{1 \leq m_{k} \leq d ; \\ m_{k} \neq l_{k}}} \frac{\left|V\left(\tau_{1}, \ldots, \tau_{d}\right)\right|}{\prod_{1 \leq k \leq\left|\mathfrak{I}_{2}\right|, l_{k} \in \mathfrak{I}_{2}}\left|\tau_{l_{k}}-\tau_{m_{k}}\right|} .
$$

Evaluating this at $\tau_{l_{k}}=\zeta_{l_{k}}$ for $l_{k} \in \mathfrak{I}_{2}$, we have $\left|\tau_{l_{k}}-\tau_{m_{k}}\right|=\left|\zeta_{l_{k}}-\tau_{m_{k}}\right|$, or $\left|\tau_{l_{k}}-\tau_{m_{k}}\right|=$ $\left|\zeta_{l_{k}}-\zeta_{m_{k}}\right|$ if $m_{k} \in \mathfrak{I}_{2}$. Since $\tau_{l}$ for $l \in[i, d]$ are free indices with bound indices, $l$ and $m(\neq l) \in[1, d]$ are in different bands so that $\left|\tau_{l}-\tau_{m}\right|>\delta \alpha_{1}^{\frac{1}{2}}\left|\tau_{l} \tau_{m}\right|^{-\frac{K}{d(d+1)}}$. Then, by the fact that $\left|\tau_{l}-\zeta_{l}\right| \leq\left|u_{j(l)}\right| \lesssim \varepsilon \delta\left|\tau_{l}\right|$ for $l \in[i, d]$, we see that $\left|\zeta_{l}-\zeta_{m}\right| \sim\left|\tau_{l}-\tau_{m}\right|$ for $i \leq l \leq d$ and $1 \leq m \leq d$ if $\varepsilon$ is sufficiently small. Similarly, we also have $\left|\zeta_{l}-\tau_{m}\right| \sim\left|\tau_{l}-\tau_{m}\right|$. So $\left|V\left(\tau_{1}, \ldots, \tau_{d}\right)\right|$ at $\tau_{l}=\zeta_{l}, l \in \mathfrak{I}_{2}$ can be bounded above by $\left|V\left(\tau_{1}, \ldots, \tau_{d}\right)\right|$.

Now we investigate lower bounds of $\left|\tau_{l}-\tau_{m}\right|$ for $l \in \mathfrak{I}_{2}$ and $1 \leq m \leq d$. If $\left|\tau_{l}\right| \ll\left|\tau_{m}\right|$ or $\left|\tau_{m}\right| \ll\left|\tau_{l}\right|$, it follows from (22) that

$$
\left|\tau_{l}-\tau_{m}\right| \gtrsim\left|\tau_{l}\right| \gtrsim \alpha_{1}^{\frac{1}{2}}\left|\tau_{l}\right|^{-\frac{2 K}{d(d+1)}}
$$

If $\left|\tau_{l}\right| \sim\left|\tau_{m}\right|$, (ii) in Lemma 11 implies that

$$
\left|\tau_{l}-\tau_{m}\right| \gtrsim \delta \alpha_{1}^{\frac{1}{2}}\left|\tau_{l} \tau_{m}\right|^{-\frac{K}{d(d+1)}} \sim \delta \alpha_{1}^{\frac{1}{2}}\left|\tau_{l}\right|^{-\frac{2 K}{d(d+1)}} .
$$

So we get

$$
\prod_{1 \leq k \leq\left|\Im_{2}\right|, l_{k} \in \Im_{2}}\left|\tau_{l_{k}}-\tau_{m_{k}}\right| \gtrsim \prod_{l \in \Im_{2}} \delta \alpha_{1}^{\frac{1}{2}}\left|\tau_{l}\right|^{-\frac{2 K}{d(d+1)}} .
$$


By (30), we obtain that

$$
\begin{aligned}
|I| & \lesssim \max _{1 \leq i \leq d}\left|\tau_{i}\right|^{M-d}\left|V\left(\tau_{1}, \ldots, \tau_{d}\right)\right| \frac{\prod_{l=i}^{d}\left|u_{j(l)}\right|}{\prod_{l \in \mathfrak{I}_{1}}\left|\tau_{l}\right| \prod_{l \in \mathfrak{I}_{2}} \delta \alpha_{1}^{\frac{1}{2}}\left|\tau_{l}\right|^{-\frac{2 K}{d(d+1)}}} \\
& \lesssim\left|J_{d}(\tau)\right| \prod_{l \in \mathfrak{I}_{1}} \frac{\varepsilon \delta\left|\tau_{l}\right|}{\left|\tau_{l}\right|} \prod_{l \in \mathfrak{I}_{2}} \frac{\varepsilon \delta \alpha_{1}^{\frac{1}{2}}\left|\tau_{l}\right|^{-\frac{2 K}{d(d+1)}}}{\delta \alpha_{1}^{\frac{1}{2}}\left|\tau_{l}\right|^{-\frac{2 K}{d(d+1)}}} \\
& \lesssim \varepsilon^{d-i+1}\left|J_{d}(\tau)\right| .
\end{aligned}
$$

Hence the error terms $I$ and $I I$ are bounded by $\left|J_{d}(\tau)\right| \cdot O(\varepsilon)$.

If we set $E_{1}=E_{2}$ and $\alpha_{1}=\alpha_{2}$ in Proposition 1, then $T$ is of restricted weak type for $\left(p_{d}, q_{d}\right)$. Moreover, the weak type estimate can be obtained by the argument in [11]. (See also [13].)

Proposition 2 Let $F$ be a (Borel) measurable set. For $f \in L^{p_{d}, u}$, there exists a constant $C>0$, depending only on $p_{d}, q_{d}, u$, such that

$$
\left\langle T f, \chi_{F}\right\rangle \leq C\|f\|_{L^{p_{d}, u}|F|^{\frac{1}{q_{d}^{\prime}}}} .
$$

Here, $|F|$ is the Lebesgue measure of $F$ on $\mathbb{R}^{2 d}$ and $1 / q_{d}^{\prime}=1-1 / q_{d}, u<q_{d}$.

Let us set $f=\sum_{k=-\infty}^{\infty} 2^{k} \chi_{E_{k}}$ where the $E_{k}$ 's are pairwise disjoint measurable sets in $\mathbb{R}^{2 d}$. The key idea is controlling interaction between the sets $E_{k}$ 's, which is shown in Proposition 1. In fact, the Christ's argument in [11] enables us to obtain some Lorentz space bound.

\section{Near-optimal Lorentz space inequalities}

By the argument in "Appendix" of [33], Propositions 1 and 3 imply Theorem 1.

Proposition 3 For $d \geq 3$, let $F_{1}, F_{2}, E \subset \mathbb{R}^{2 d}$ be measurable sets with finite measure. Suppose that

$$
T^{*} \chi_{F_{1}}(y) \geq \beta_{1} \text { and } T^{*} \chi_{F_{2}}(y) \geq \beta_{2}
$$

for all $y \in E$ and $\beta_{1} \leq \beta_{2}$. Suppose that $\alpha_{i}$ for $i=1,2$ such that $\left\langle T^{*} \chi_{F_{i}}, \chi_{E}\right\rangle /\left|F_{i}\right| \geq \alpha_{i}$ and $\alpha_{2} \leq \alpha_{1}$. Then there exists a constant $C>0$, depending on $N$ and $d$, such that

$$
\left|F_{2}\right| \geq C \alpha_{1}^{r_{1}} \alpha_{2}^{r_{2}} \beta_{1}^{s_{1}} \beta_{2}^{s_{2}}
$$

where $r_{1}+r_{2}=\frac{d(d-1)}{2}, s_{1}+s_{2}=d$, and $\frac{s_{2}}{q_{d}^{\prime}}-\frac{r_{2}}{q_{d}}-1>0$.

The main idea of the proof of Proposition 3 is 'the two-stage band structure.' Stovall also showed how to handle the polynomial curves with the affine arclength measure in [34]. Here we provide a sketch of the proof.

Instead of $2 d$ variables in Sect. 3, we consider $2 d-1$ variables. Then we can obtain analogues of Lemmas 8 and 9 , where $\alpha_{2}$ corresponding to $z_{2 d}$ should be replaced by $\beta_{2}$ with $z_{2 d-1}$.

We consider various cases. If $\beta_{1} \gtrsim \alpha_{1}$, Proposition 3 can be deduced easily by the same proof of Lemma 10. 
Now we assume that $\beta_{1} \ll \alpha_{1}$. By the same argument in the proof of Lemma 11, we obtain a band structure on $\{2 d-k, \ldots, 2 d-1\}$ and a set $\omega \subset \mathbb{C}^{k}$ with

$$
\sigma(\omega) \sim \alpha_{1}^{\left\lfloor\frac{k}{2}\right\rfloor} \beta_{1}^{\left\lceil\frac{k}{2}\right\rceil}\left(\frac{\beta_{2}}{\beta_{1}}\right),
$$

and (i)-(iv) of Lemma 11. If we assume $\beta_{2} \gtrsim \alpha_{1}$, then $2 d-1$ must be a free index without quasi-bound and bound indices. By slight modification of (29) and by (35), it follows that

$$
\begin{aligned}
\left|F_{2}\right| & \gtrsim \alpha_{1}^{\frac{d(d-1)}{2}}\left(\frac{\beta_{1}}{\alpha_{1}}\right)^{\mathcal{N}}\left(\frac{\beta_{2}}{\alpha_{1}}\right)^{d-1} \alpha_{1}^{d-k} \alpha_{1}^{\left\lfloor\frac{k}{2}\right\rfloor} \beta_{1}^{\left\lceil\frac{k}{2}\right\rceil}\left(\frac{\beta_{2}}{\beta_{1}}\right) \\
& =\alpha^{\frac{d(d+1)}{2}}\left(\frac{\beta_{1}}{\alpha_{1}}\right)^{\mathcal{N}+\left\lceil\frac{k}{2}\right\rceil}\left(\frac{\beta_{2}}{\alpha_{1}}\right)^{d-1}\left(\frac{\beta_{2}}{\beta_{1}}\right) .
\end{aligned}
$$

Since $2 d-k, 2 d-1$, and all even indices between $2 d-k$ and $2 d-1$ are free indices, the number of free indices is at least $\left\lfloor\frac{k}{2}\right\rfloor+2$. Hence $\mathcal{N}+\left\lceil\frac{k}{2}\right\rceil \leq d-1$. Since $\beta_{2} \gtrsim \alpha_{1}$, we conclude that $\left|F_{2}\right| \gtrsim \alpha_{1}^{\frac{d(d-1)}{2}} \beta_{1}^{d-2} \beta_{2}^{2}$. This satisfies the relations in Proposition 3 .

Now we assume that $\beta_{2} \ll \alpha_{1}$. Then the index $2 d-1$ may not be free. The number of free indices is at least $\left\lfloor\frac{k}{2}\right\rfloor+1$, which means $\mathcal{N}+\left\lceil\frac{k}{2}\right\rceil \leq d$. One can see that this is not enough for the desired bound. So we follow the argument of the two-stage band structure in [33].

Let $\mathcal{B}(2 d-1)$ be the band containing $2 d-1$ of $\{2 d-k, \ldots, 2 d-1\}$. Now, we decompose $\mathcal{B}(2 d-1)$ into sub-bands as follows. For $\varepsilon>0$, there exist $\rho$ and $\rho^{\prime}$ such that $c_{d, \varepsilon}<\rho^{\prime}<$ $\varepsilon \rho<\delta^{\prime}$, and a subset $\omega^{\prime}$ of $\omega$ satisfying that $\sigma\left(\omega^{\prime}\right) \sim \sigma(\omega)$. Then the following properties hold:

(i) $\left|z_{i}-z_{j}\right|>\rho \gamma_{2}^{1 / 2}\left|z_{i} z_{j}\right|^{-K / d(d+1)}$ unless $i$ and $j$ lie in the same band.

(ii) $c_{0} \beta_{1}^{1 / 2}\left|z_{i} z_{j}\right|^{-K / d(d+1)}<\left|z_{i}-z_{j}\right| \leq \rho \gamma_{2}^{1 / 2}\left|z_{i} z_{j}\right|^{-K / d(d+1)}$ whenever $i$ is quasi-bound to $j$.

(iii) $\rho^{\prime} \gamma_{2}^{1 / 2}\left|z_{i} z_{j}\right|^{-K / d(d+1)}>\left|z_{i}-z_{j}\right|$ whenever $i$ is bound to $j$,

for $i, j \in \mathcal{B}(2 d-1), \gamma_{2}=\max \left\{\alpha_{2}, \beta_{2}\right\}$ and some constant $c_{0}>0$.

Then the number of free and quasi-free indices in $\{2 d-k, \ldots, 2 d-1\}$ may increase. So we repeat discarding indices as in the proof of Lemma 11 until we get exactly $d$ free and quasi-free indices in $\left\{2 d-k^{\prime}, \ldots, 2 d-1\right\}$ for some integer $k^{\prime}$. By abuse of notation, we will write $k$ instead of $k^{\prime}$.

Let $F_{1}$ and $Q_{1}$ be the number of free and quasi-free indices which are contained in $\{2 d-k, \ldots, 2 d-1\} \backslash \mathcal{B}(2 d-1)$. Also, let $F_{2}$ and $Q_{2}$ be the number of free and quasi-free indices in sub-bands of $\mathcal{B}(2 d-1)$. Note that $F_{1}+Q_{1}+F_{2}+Q_{2}=d$. We set $M=F_{2}+Q_{2}$ and denote by $N$ the number of elements of $\mathcal{B}(2 d-1)$. Then $N-M$ denotes the number of bound indices in sub-bands of $\mathcal{B}(2 d-1)$.

The case when $\mathcal{B}(2 d-1)=2 d-1$ can be treated as in the case $\beta_{2} \gtrsim \alpha_{1}$ above. Hence it suffices to consider the following three cases:

- $2 d-1$ is free and there is at least one free index other than $2 d-1$ in $\mathcal{B}(2 d-1)$.

- $2 d-1$ is quasi-free.

- $2 d-1$ is bound to some $j$ in $\mathcal{B}(2 d-1)$.

Each case is managed by a similar computation in [33]. We omit the details. 


\section{Proof of Theorem 2}

This section seems to be redundant,

Let $\epsilon$ be sufficiently small so that Lemmas 6 and 7 hold. As in the beginning of Sect. 3, it is enough to consider

$$
T f(x)=\int_{\Delta} f(x-\Gamma(z))\left|\tau_{\Gamma}(z)\right|^{\frac{1}{3}} \mathrm{~d} \mu(z) \sim \int_{\Delta} f(x-\Gamma(z))|z|^{\frac{K}{3}} \mathrm{~d} \mu(z),
$$

where $K=a_{1}+a_{2}+a_{3}-6 \geq 0$ and $\Delta=\Delta_{0}$ in Lemma 7. We set $\mathrm{d} \sigma(z)=|z|^{K / 3} \mathrm{~d} \mu(z)$.

By the argument in "Appendix" in [33], the following two lemmas imply Theorem 2.

Lemma 13 Let $E_{1}, E_{2}, G \subset \mathbb{R}^{6}$ be measurable sets with finite measure. Suppose that $T \chi_{E_{1}}(x) \geq \alpha_{1}$ and $T \chi_{E_{2}}(x) \geq \alpha_{2}$ for all $x \in G$ and $\alpha_{2} \geq \alpha_{1}$. Then

$$
\left|E_{2}\right| \gtrsim \alpha_{1}^{2-2 v} \beta^{2} \alpha_{2}^{2+2 v}
$$

where $\beta=\alpha_{1}|F| /\left|E_{1}\right|$ and $v=3 /(K+6)$.

Lemma 14 Let $F_{1}, F_{2}, E \subset \mathbb{R}^{6}$ be measurable sets with finite measure. Suppose that $T^{*} \chi_{F_{1}}(y) \geq \beta_{1}$ and $T^{*} \chi_{F_{2}}(x) \geq \beta_{2}$ for all $y \in E$ and $\beta_{1} \leq \beta_{2}$. Then

$$
\left|F_{2}\right| \gtrsim \alpha^{3} \beta_{1} \beta_{2}^{2}
$$

for $\alpha=\beta_{1}|E| /\left|F_{1}\right|$.

Proof of Lemma 13 By Lemmas 8, 9 and Step 1 in the proof of Lemma 11, there exist subsets $P_{i}$ for $1 \leq i \leq 6$ of $\Delta$ such that (18), (19), (23), and (24) holds for $d=3$. In addition to (23), we have

$$
\left|z_{6}-z_{i}\right| \gtrsim \alpha_{2}^{\frac{1+2 v}{4}} \alpha_{1}^{\frac{1-2 v}{4}}\left|z_{i} z_{6}\right|^{-\frac{K}{12}} \text { for } i<6 .
$$

If $\left|z_{i}\right| \sim\left|z_{6}\right|$, then (38) follows by (iii) in Lemma 9 and the fact that $\alpha_{2} \geq \alpha_{1}$ and $v \leq 1 / 2$. If $\left|z_{i}\right| \ll\left|z_{6}\right|$ or $\left|z_{i}\right| \gg\left|z_{6}\right|$, then $\left|z_{6}-z_{i}\right| \gtrsim\left|z_{6}\right|=\left|z_{6}\right|^{1+\frac{K}{12}}\left|z_{i}\right|^{\frac{K}{12}}\left|z_{i} z_{6}\right|^{-\frac{K}{12}}$. Since $\gamma \geq \alpha_{1}$, (38) follows by (19).

Following the proof of Lemma 10 for $d=3$, we obtain

$$
\left|E_{2}\right| \gtrsim \int_{\Phi} \prod_{i=4}^{6}\left|z_{i}\right|^{\frac{2 K}{3}} \prod_{4 \leq i<j \leq 6}\left|z_{j}-z_{i}\right|^{2} \mathrm{~d} \mu(z)
$$

by (11). Then (23) and (38) give

$$
\begin{gathered}
\left|E_{2}\right| \gtrsim \alpha_{1}^{1-2 v} \beta \alpha_{2}^{1+2 v} \int_{\Phi} \prod_{i=4}^{6}\left|z_{i}\right|^{\frac{2 K}{3}}\left|z_{4} z_{5}\right|^{-\frac{K}{6}}\left|z_{4} z_{6}\right|^{-\frac{K}{6}}\left|z_{5} z_{6}\right|^{-\frac{K}{6}} \mathrm{~d} \mu(z) \\
\sim \alpha_{1}^{1-2 v} \beta \alpha_{2}^{1+2 v} \sigma^{3}(\Phi) \sim \alpha_{1}^{2-2 v} \beta^{2} \alpha_{2}^{2+2 v} .
\end{gathered}
$$

This completes the proof.

Interchanging the roll of $\alpha$ and $\beta$ in the proofs of Lemmas 8 and 9, we obtain the following.

Lemma 15 Let $v=3 /(K+6)$. Suppose that the assumption of Proposition 14 holds and let $\gamma=\max \left\{\beta_{1}, \alpha\right\}$. Then there exists a sequence of subsets $P_{1}, P_{2}, P_{3}$ of $\Delta$ such that $\sigma\left(P_{1}\right) \gtrsim \beta_{1}, \sigma\left(P_{2}\right) \gtrsim \alpha$, and $\sigma\left(P_{3}\right) \gtrsim \beta_{2}$, where the implicit constants depend only on 
d. Also there exists constants $c_{v}>0$ and c such that on $P_{j},\left|z_{j}\right| \geq c_{\nu} \gamma^{\nu}$ for $1 \leq j \leq 2$, $\left|z_{3}\right| \geq c_{\nu} \beta_{2}^{v}$, and

$$
\left|z_{2}-z_{1}\right| \geq c \alpha^{\frac{1}{2}}\left|z_{1} z_{2}\right|^{-\frac{K}{12}}, \quad\left|z_{3}-z_{i}\right| \geq \begin{cases}c \beta_{2}^{\frac{1}{2}}\left|z_{3}\right|^{-\frac{K}{6}} & \text { if }\left|z_{i}\right|<\frac{1}{2} c_{\nu} \beta_{2}^{v} \\ c \beta_{2}^{\frac{1}{2}}\left|z_{i}\right|^{-\frac{K}{6}} & \text { if }\left|z_{i}\right| \geq \frac{1}{2} c_{\nu} \beta_{2}^{v} .\end{cases}
$$

Applying Lemma 15 to the proof of Lemma 13, we may get $|F| \gtrsim \alpha^{2} \beta_{1}^{2-2 v} \beta_{2}^{2+2 v}$, which is not enough to establish the optimal Lorentz space estimates. So we separate cases according to the size of $\alpha$ and $\beta_{1}$.

Proof of Lemma 14 As in the proof of Lemma 13, we have

$$
\left|F_{2}\right| \gtrsim \int_{P_{1}} \int_{P_{2}} \int_{P_{3}} \prod_{i=1}^{3}\left|z_{i}\right|^{\frac{2 K}{3}} \prod_{1 \leq i<j \leq 3}\left|z_{j}-z_{i}\right|^{2} \mathrm{~d} \mu\left(z_{3}\right) \mathrm{d} \mu\left(z_{2}\right) \mathrm{d} \mu\left(z_{1}\right) .
$$

Now we consider two cases: $\beta_{1} \gtrsim \alpha$ or $\beta_{1} \ll \alpha$.

Case $1 \beta_{1} \gtrsim \alpha$. As in the proof of Lemma 13, we obtain by Lemma 15

$$
\left|z_{3}-z_{i}\right| \geq c \beta_{2}^{\frac{1+2 v}{4}} \beta_{1}^{\frac{1-2 v}{4}}\left|z_{i} z_{3}\right|^{-\frac{K}{12}} .
$$

Then

$$
\left|F_{2}\right| \gtrsim \alpha \beta_{2}^{1+2 v} \beta_{1}^{1-2 v} \prod_{i=1}^{3} \sigma\left(P_{i}\right) \gtrsim \alpha^{2} \beta_{2}^{2+2 v} \beta_{1}^{2-2 v} \gtrsim \alpha^{3} \beta_{2}^{2} \beta_{1} .
$$

The last inequality follows by the fact that $\alpha \lesssim \beta_{1} \leq \beta_{2}$.

Case $2 \beta_{1} \ll \alpha$. Since $\left|z_{2}-z_{1}\right| \geq c \alpha^{\frac{1}{2}}\left|z_{1} z_{2}\right|^{-\frac{K}{12}}$, we consider two disjoint balls which are defined by

$$
B_{\delta, \alpha}\left(z_{i}\right)=\left\{z:\left|z-z_{i}\right|<\delta \alpha^{\frac{1}{2}}\left|z_{1} z_{2}\right|^{-\frac{K}{12}}\right\}, \quad \text { for } i=1,2
$$

where we choose $\delta=\min \left\{c / 3, c_{v}^{K / 6+1} / 2\right\}$. Then $z_{3}$ can be located in $B_{\delta, \alpha}\left(z_{1}\right), B_{\delta, \alpha}\left(z_{2}\right)$, or $\left(B_{\delta, \alpha}\left(z_{1}\right) \cup B_{\delta, \alpha}\left(z_{2}\right)\right)^{c}$.

If $z_{3} \in B_{\delta, \alpha}\left(z_{1}\right)$, then $z_{3} \notin B_{\delta, \alpha}\left(z_{2}\right)$, i.e., $\left|z_{3}-z_{2}\right| \geq \delta \alpha^{\frac{1}{2}}\left|z_{1} z_{2}\right|^{-\frac{K}{12}}$. Since $\left|z_{1}\right|,\left|z_{2}\right| \geq$ $c_{\nu} \alpha^{v}$, we have $\left|z_{3}-z_{1}\right|<\delta \alpha^{\frac{1}{2}}\left|z_{1} z_{2}\right|^{-\frac{K}{12}} \leq \delta c_{v}^{-K / 6} \alpha^{v} \leq \delta c_{v}^{-K / 6-1}\left|z_{1}\right| \leq\left|z_{1}\right| / 2$. So $\left|z_{1}\right| / 2 \leq\left|z_{3}\right| \leq 3\left|z_{1}\right| / 2$, and then $\left|z_{3}-z_{2}\right| \geq \delta \alpha^{\frac{1}{2}}\left|z_{2} z_{3}\right|^{-\frac{K}{12}}$. Also we obtain $\left|z_{3}-z_{1}\right| \gtrsim$ $\beta_{2}^{\frac{1}{2}}\left|z_{1} z_{3}\right|^{-\frac{K}{12}}$ by Lemma 15 .

Hence (39) implies that

$$
\left|F_{2}\right| \gtrsim \alpha^{2} \beta_{2} \int_{P_{1}} \int_{P_{2}} \int_{P_{3}} \prod_{i=1}^{3}\left|z_{i}\right|^{\frac{2 K}{3}}\left|z_{1} z_{2} z_{3}\right|^{-\frac{K}{3}} \mathrm{~d} \mu\left(z_{3}\right) \mathrm{d} \mu\left(z_{2}\right) \mathrm{d} \mu\left(z_{1}\right) \gtrsim \alpha^{3} \beta_{1} \beta_{2}^{2} .
$$

The case of $z_{3} \in B_{\delta, \alpha}\left(z_{2}\right)$ can be treated similarly.

If $z_{3} \in\left(B_{\delta, \alpha}\left(z_{1}\right) \cup B_{\delta, \alpha}\left(z_{2}\right)\right)^{c}$, we have $\left|z_{3}-z_{1}\right| \geq \delta \alpha^{\frac{1}{2}}\left|z_{1} z_{2}\right|^{-\frac{K}{12}}$ and $\left|z_{3}-z_{2}\right| \geq$ $\delta \alpha^{\frac{1}{2}}\left|z_{1} z_{2}\right|^{-\frac{K}{12}}$.

In this case, we consider the sub-cases according to the size of $\left|z_{1}\right|$ and $\left|z_{3}\right|$.

- $\left|z_{3}\right| \sim\left|z_{1}\right|$ : We get $\left|z_{3}-z_{2}\right| \gtrsim \delta \alpha^{\frac{1}{2}}\left|z_{2} z_{3}\right|^{-\frac{K}{12}}$. Also $\left|z_{3}-z_{1}\right| \gtrsim \beta_{2}^{\frac{1}{2}}\left|z_{1} z_{3}\right|^{-\frac{K}{12}}$ by Lemma 15. So, $\left|F_{2}\right| \gtrsim \alpha^{2} \beta_{2} \sigma\left(P_{1}\right) \sigma\left(P_{2}\right) \sigma\left(P_{3}\right) \gtrsim \alpha^{3} \beta_{2}^{2} \beta_{1}$. 
- $\left|z_{3}\right| \ll\left|z_{1}\right|$ or $\left|z_{1}\right| \ll\left|z_{3}\right|:$

We get $\left|z_{3}-z_{1}\right| \gtrsim\left|z_{3}\right|$. Since $v=3 /(K+6)$ and $\left|z_{3}\right| \geq c_{\nu} \beta_{2}^{v}$, we obtain

$$
\begin{aligned}
\left|F_{2}\right| & \gtrsim \alpha^{2} \int_{P_{1}} \int_{P_{2}} \int_{P_{3}} \prod_{i=1}^{3}\left|z_{i}\right|^{\frac{2 K}{3}}\left|z_{1} z_{2}\right|^{-\frac{K}{3}}\left|z_{3}\right|^{2} \mathrm{~d} \mu\left(z_{3}\right) \mathrm{d} \mu\left(z_{2}\right) \mathrm{d} \mu\left(z_{1}\right) \\
& \gtrsim \alpha^{2} \iiint \prod_{i=1}^{3}\left|z_{i}\right|^{\frac{K}{3}}\left|z_{3}\right|^{\frac{1}{v}} \mathrm{~d} \mu\left(z_{3}\right) \mathrm{d} \mu\left(z_{2}\right) \mathrm{d} \mu\left(z_{1}\right) \\
& \gtrsim \alpha^{2} \beta_{2} \sigma\left(P_{1}\right) \sigma\left(P_{2}\right) \sigma\left(P_{3}\right) \gtrsim \alpha^{3} \beta_{1} \beta_{2}^{2} .
\end{aligned}
$$

This completes the proof.

Acknowledgements The authors thank anonymous referees for pointing out the reference [36] and for many helpful comments and suggestions which greatly improve the presentation of our results.

\section{A The necessary conditions}

The necessary conditions can be obtained by a straightforward adaptation of the counterexamples from [10,33].

Let $\mathcal{A} f(x)=\int_{D} f(x-\Gamma(z)) \mathrm{d} \mu(z)$ for $\Gamma(z)=\left(z, z^{2}, \ldots, z^{d-1}, z^{d}\right)$. Also let $D_{r}$ be an anisotropic scaling in $\mathbb{R}^{2 d}$ given by

$$
D_{r}\left(x_{1}, y_{1}, x_{2}, y_{2}, \ldots, x_{d}, y_{d}\right)=\left(r x_{1}, r y_{1}, r^{2} x_{2}, r^{2} y_{2}, \ldots, r^{d} x_{d}, r^{d} y_{d}\right) .
$$

We denote by $B(x, \varepsilon)$ a ball of radius $\varepsilon$ centered at $x$ in $\mathbb{R}^{2 d}$ and by $N(x, \varepsilon)$ the $\varepsilon$ neighborhood of $-\Gamma(z)$ translated by $x$.

The restricted weak type. Using the dilation of $z \mapsto r z$ for $0<r<1$, we have

$$
\mathcal{A} \chi_{D_{r} N(0,1)}(y) \gtrsim r^{2} \chi_{D_{r} B(0,1)}(y) \text {. }
$$

Since $\left|D_{r} N(0,1)\right|^{1 / p} \sim r^{d(d+1) / p}$, the restricted weak type $(p, q)$ implies $r^{2+d(d+1) / q} \lesssim$ $r^{d(d+1) / p}$. It follows that $1+d(d+1) / 2 q \geq d(d+1) / 2 p$.

Considering $N(0, \varepsilon)$ instead of $D_{r} N(0,1)$, we can also obtain $d / q \geq(d-1) / p$. By duality, $1+(d-1) / q \geq d / p$ follows.

Finally, the condition $q \geq p$ holds by the fact that $\mathcal{A}$ is translation invariant. In fact, if any nonzero linear operator which is translation invariant is bounded from $L^{p}\left(\mathbb{R}^{d}\right)$ to $L^{q}\left(\mathbb{R}^{d}\right)$, then $q \geq p$ is necessary. (See Section 2.5.3 in [21].)

As a result, if $\mathcal{A}$ is of restricted weak type $(p, q)$, then $(p, q)$ must be in $\mathcal{R}$, where $\mathcal{R}$ is a trapezoid with vertices $(0,0),(1,1),\left(1 / p_{d}, 1 / q_{d}\right)$, and $\left(1-1 / q_{d}, 1-1 / p_{d}\right)$.

The endpoints Now we show that if (2) holds, then $u \leq q_{d}, p_{d} \leq v$, and $u \leq v$, where $\left(p_{d}, q_{d}\right)=\left(\frac{d+1}{2}, \frac{d(d+1)}{2(d-1)}\right)$.

Let $M \geq 2$ be a positive integer. For given $\eta_{j}$ and $r_{j}, j=1, \ldots, M$, we can choose $x_{j}$ such that $D_{r_{j}} B\left(x_{j}, \eta_{j}\right)$ are pairwise disjoint and $D_{r_{j}} N\left(x_{j}, \eta_{j}\right)$ are also pairwise disjoint. (The choice of $\eta_{j}$ and $r_{j}$ will be borrowed from Section 3 in [33].)

- $u \leq v$ : Choose $\eta_{j}=\varepsilon^{j}$ for $0<\varepsilon<1$ and $r_{j}=1$. Let

$$
f(y)=\sum_{j=1}^{M} \varepsilon^{-2(d-1) j / p_{d}} \chi_{N\left(x_{j}, \varepsilon^{j}\right)}(y)
$$


so that $\|f\|_{L^{p_{d}, u}} \sim M^{\frac{1}{u}}$. Since $\mathcal{A} \chi_{N\left(x_{j}, \varepsilon^{j}\right)}(y) \gtrsim \chi_{B\left(x_{j}, \varepsilon^{j}\right)}(y)$ and $B\left(x_{j}, \varepsilon^{j}\right)$ are pairwise disjoint, we obtain that $\|\mathcal{A} f\|_{L^{q_{d}, v}} \gtrsim M^{\frac{1}{v}}$. Therefore (2) gives $M^{1 / v} \lesssim M^{1 / u}$ for any positive integer $M \geq 2$. Hence we obtain $u \leq v$.

- $u \leq q_{d}$ : By the definition of $D_{r} N(x, \varepsilon)$, we see that $\mathcal{A} \chi_{D_{r} N(x, \varepsilon)}(y) \geq r^{2} \chi_{D_{r} B(x, \varepsilon)}(y)$ for any $x$ and $0<\varepsilon, r<1$.

Let $\varepsilon_{j}=2^{-(M-j) p_{d}}, r_{j}=2^{-j}$. Considering $f=\sum_{j=1}^{M} 2^{2 j} \chi_{D_{r_{j}} N\left(x_{j}, \varepsilon_{j}\right)}$, we get $\|f\|_{L^{p_{d}, u}} \sim M^{1 / u} 2^{-2 M(d-1)}$. Also, we have

$\|\mathcal{A} f\|_{L^{q_{d}, v}} \gtrsim\left\|\sum_{j=1}^{M} 2^{2 j} 2^{-2 j} \chi_{D_{r_{j}} B\left(x_{j}, \varepsilon_{j}\right)}\right\|_{L^{q_{d}, v}}=\left|\bigcup_{j=1}^{M} D_{r_{j}} B\left(x_{j}, \varepsilon_{j}\right)\right|^{\frac{1}{q_{d}}}=M^{\frac{1}{q_{d}}} 2^{-\frac{2 d M_{p_{d}}}{q_{d}}}$.

Thus (2) implies that $M^{1 / q_{d}} 2^{-2 d M p_{d} / q_{d}} \lesssim M^{1 / u} 2^{-2 M(d-1)}$. Since $2^{2 p_{d} M\left(d / q_{d}-(d-1) / p_{d}\right)}$ $=1$, we get $u \leq q_{d}$ whenever $M \geq 2$.

- $p_{d} \leq v$ : In this case, we make use of $\mathcal{A}^{*} \chi_{D_{r} B(x, \varepsilon)} \gtrsim \varepsilon^{2} r^{2}$ on $D_{r} N(x, \varepsilon)$, which can be obtained by the same calculation as above and by $\varepsilon^{2}<1$. For $-M \leq j \leq-1$, we set $\varepsilon_{j}=2^{-(j+M) q_{d}^{\prime}}, r_{j}=2^{j q_{d}^{\prime} / q_{d}}$ and $f=\sum_{i=-1}^{-M} 2^{2 j} \chi_{D_{r_{j}} B\left(x_{j}, \varepsilon_{j}\right)}$. If (2) holds, its dual estimate implies $M^{\frac{1}{p_{d}^{\prime}}} 2^{-2 M q_{d}^{\prime}\left(1+\frac{d-1}{p_{d}^{\prime}}\right)} \lesssim M^{1 / v^{\prime}} 2^{-2 d M}$. Since $1+\frac{d-1}{p_{d}^{\prime}}=\frac{d}{q_{d}^{\prime}}$, it follows that $v^{\prime} \leq p_{d}^{\prime}$ for any positive integer $M \geq 2$.

\section{References}

1. Bak, J.-G., Ham, S.: Restriction of the Fourier transform to some complex curves. J. Math. Anal. Appl. 409, 1107-1127 (2014)

2. Bak, J.-G., Oberlin, D., Seeger, A.: Two endpoint bounds for generalized Radon transforms in the plane. Rev. Mat. Iberoamericana. 18, 231-247 (2002)

3. Bak, J.-G., Oberlin, D., Seeger, A.: Restriction of Fourier transforms to curves and related oscillatory integrals. Am. J. Math. 131, 277-311 (2009)

4. Bak, J.-G., Oberlin, D., Seeger, A.: Restriction of Fourier transforms to curves, II: some classes with vanishing torsion. J. Aust. Math. Soc. 85, 1-28 (2008)

5. Bak, J.-G., Oberlin, D., Seeger, A.: Restriction of Fourier transforms to curves: an endpoint estimate with affine arclength measure. J. Reine Angew. Math. 682, 167-205 (2013)

6. Bennett, J., Seeger, A.: The Fourier extension operator on large spheres and related oscillatory integrals. Proc. Lond. Math. Soc. 98, 45-82 (2009)

7. Choi, Y.: Convolution operators with the affine arclength measure on plane curves. J. Korean Math. Soc. 36, 193-207 (1999)

8. Choi, Y.: The $L^{p}-L^{q}$ mapping properties of convolution operators with the affine arclength measure on space curves. J. Aust. Math. Soc. 75, 247-261 (2003)

9. Christ, M.: On the restriction of the Fourier transform to curves: endpoint results and the degenerate case. Trans. Am. Math. Soc. 287, 223-238 (1985)

10. Christ, M.: Convolution, curvature, and combinatorics: a case study. Int. Math. Res. Not. 19, 1033-1048 (1998)

11. Christ, M.: Quasi-extremals for a Radon-like transform. Preprint arXiv:1106.0722 (2011)

12. Dendrinos, S.: Generalisations of an inequality of Hardy under polynomial changes of variables. Ph.D. thesis, University of Edinburgh (2005)

13. Dendrinos, S., Laghi, N., Wright, J.: Universal $L^{p}$ improving for averages along polynomial curves in low dimensions. J. Funct. Anal. 257, 1355-1378 (2009)

14. Dendrinos, S., Müller, D.: Uniform estimates for the local restriction of the Fourier transform to curves. Trans. Am. Math. Soc. 365, 3477-3492 (2013)

15. Dendrinos, S., Stovall, B.: Uniform bounds for convolution and restricted X-ray transforms along degenerate curves. J. Funct. Anal. 268, 585-633 (2015) 
16. Dendrinos, S., Wright, J.: Fourier restriction to polynomial curves I : a geometric inequality. Am. J. Math. 132, 1031-1076 (2010)

17. Drury, S.: Degenerate curves and harmonic analysis. Math. Proc. Camb. Philos. Soc. 108, 89-96 (1990)

18. Drury, S., Guo, K.: Convolution estimates related to surfaces of half the ambient dimension. Math. Proc. Camb. Philos. Soc. 110, 151-159 (1991)

19. Drury, S., Marshall, B.: Fourier restriction theorems for curves with affine and Euclidean arclengths. Math. Proc. Camb. Philos. Soc. 97, 111-125 (1985)

20. Drury, S., Marshall, B.: Fourier restriction theorems for degenerate curves. Math. Proc. Camb. Philos. Soc. 101, 541-553 (1987)

21. Grafakos, L.: Classical Fourier Analysis. Graduate Texts in Mathematics, p. 249. Springer, Berlin (2008)

22. Gressman, P.: Convolution and fractional integration with measures on homogeneous curves in $\mathbb{R}^{n}$. Math. Res. Lett. 11, 869-881 (2004)

23. Ham, S., Lee, S.: Restriction estimates for space curves with respect to general measures. Adv. Math. 254, 251-279 (2014)

24. Littman, W.: $L^{p}-L^{q}$ estimates for singular integral operators arising from hyperbolic equations. Partial differential equations (Berkeley, California, 1971). In: Proceedings of Symposia in Pure Mathematics, vol. 23, pp. 479-481 (1973)

25. Oberlin, D.: Convolution estimates for some measures on curves. Proc. Am. Math. Soc. 99, 56-60 (1987)

26. Oberlin, D.: A convolution estimate for a measure on a curve in $\mathbb{R}^{4}$. Proc. Am. Math. Soc. 125, 1355-1361 (1997)

27. Oberlin, D.: Convolution with measures on curves in $\mathbb{R}^{3}$. Can. Math. Bull. 41, 478-480 (1998)

28. Oberlin, D.: A convolution estimate for a measure on a curve in $\mathbb{R}^{4}$ II. Proc. Am. Math. Soc. 127, 217-221 (1999)

29. Oberlin, D.: Convolution with measures on polynomial curves. Math. Scand. 90, 126-138 (2002)

30. Oberlin, D.: Some convolution inequalities and their applications. Trans. Am. Math. Soc. 354, 2541-2556 (2002)

31. Oberlin, D.: Convolution with measures on flat curves in low dimensions. J. Funct. Anal. 259, 1799-1815 (2010)

32. Pan, Y.: $L^{p}$-improving properties for some measures supported on curves. Math. Scand. 78, 121-132 (1996)

33. Stovall, B.: Endpoint bound for a generalized Radon transform. J. Lond. Math. Soc. 80, 357-374 (2009)

34. Stovall, B.: Endpoint $L^{p} \rightarrow L^{q}$ bounds for integration along certain polynomial curves. J. Funct. Anal. 259, 3205-3229 (2010)

35. Stovall, B.: Uniform estimates for Fourier restriction to polynomial curves in $\mathbb{R}^{d}$. Am. J. Math. 138, 449-471 (2016)

36. Weyl, H.: The Classical Groups. Princeton University Press, Princeton (1946) 